\title{
Development of theoretical approaches for post-silicon information processing
}

\author{
Viktor Ivády
}


The cover image is compiled by Viktor Ivády and licensed under the terms of the GNU Free Documentation License. The image of the Bloch sphere is created by Smite-Meister and published at https://commons.wikimedia.org/wiki/File:Bloch_sphere.svg under the GNU Free Documentation License.

(C) Viktor Ivády ISBN 978-91-7685-682-6

ISSN 0345-7524

Printed by LiU-Tryck 2016 
Családomnak 



\section{Abstract}

Despite knowing the fundamental equations in most of the physics research areas, still there is an unceasing need for theoretical method development, thanks to the more and more challenging problems addressed by the research community. The investigation of post-silicon, non-classical information processing is one of the new and rapidly developing areas that requires tremendous amount of theoretical support, new understanding, and accurate theoretical predictions.

My thesis focuses on theoretical method development for solid-state quantum information processing, mainly in the field of point defect quantum bits (qubits) in silicon carbide ( $\mathrm{SiC}$ ) and diamond. Due to recent experimental breakthroughs in this field, there are diverse theoretical problems, ranging from functional development for accurate first principles description of point defects, through complete theoretical characterization of qubits, to the modeling and simulation of actual quantum information protocols, that are needed to be addressed.

The included articles of this thesis cover the development of (i) hybrid-DFT $+\mathrm{V}_{w}$ approach for the first principles description of mixed correlated and uncorrelated systems, (ii) zero-field-splitting tensor calculation for solid-state quantum bit characterization, (iii) a comprehensive model for dynamic nuclear spin polarization of solid-state qubits in semiconductors, and (iv) group theoretical description of qubits and novel two-dimensional materials for topologically protected states. 


\section{Popular science summary}

During the last few decades, our daily life has been drastically changed by the rapid development of transistor-based digital information processing, which includes the collection, manipulation, storage, and transfer of information. Although this field is still fast evolving, the underlying technology has already faced with one of its fundamental limitations. The transistors' minimal size and thus the maximal speed are bounded by the unbreakable laws of quantum physics. The principles that limit the present "classical" information processing, however, provide a completely new alternative for communication and computation. In this approach quantum bits (qubits) store and process the information in a manner that is unachievable with transistor-based technologies. Although, the idea of such applications is known for several decades, the experimental realization is challenging.

There are several suggested alternatives for implementing qubit based information processing applications. The topic of the thesis is connected to one of the directions, in which point defects in semiconductors are considered as quantum bit realizations. At the leading edge of this research field, applicable qubit candidates, in different semiconductor host materials, such as diamond and silicon carbide, as well as simple quantum information processing protocols are investigated.

In this $\mathrm{PhD}$ thesis the development of information processing application beyond silicon-based technologies is supported by the investigation of new theoretical approaches. There are four different directions in which contributions have been made. 1) A new first principles method is designed to accurately simulate transition metal impurity related quantum bit candidates in semiconductors. 2) A theoretical tool is implemented to calculate special properties of qubits under investigation. 3) A theoretical model is introduced to describe the initialization process of nuclear quantum bits. 4) An abstract theoretical approach is used to thoroughly understand the physics of quantum bits as well as particular two-dimensional materials. 


\section{Populärvetenskaplig sammanfattning}

Under de senaste årtionden har vårt dagliga liv förändrats av den snabba utveckling inom transistorer för digital informations hantering och beräkning. Dessa applikationer utvecklas fortfarande i snabb takt men tekniken har redan kommit fram till en fundamental begränsning. Transistorers storlek, därmed deras maximala hastighet, är begränsad av de obrytbara lagar från kvantfysiken. Dessa principer, som begränsar det klassiska användandet av transistorer, har också öppnat upp för nya alternativ inom kommunikation och beräkning genom att använda kvantbitar för att lagra och hantera information på ett sätt som aldrig skulle vara möjligt med transistorer. Dessa idéer har varit kända i flera årtionden men förverkligande genom experiment har varit extremt komplicerande och utmanade.

Det finns många förslag på hur man skulle kunna implementera kvantmekaniska informationshanteringsystem. Syftet med denna avhandling är att studera lösningar baserad på halvledareteknik. Detta tillvägagångssätt har stor potential för kvantbit implementation då den kan använda de avancerade fabrikationteknik som redan finns för halvledare. I spetsen på detta forsknings område undersöks kvantbitar och enkla kvantinformationhanteringsprotokoll.

I denna doktorsavhandling introduceras nya teoretisk metoder för att bidra till utvecklingen av kvantkommunikation och beräkning bortom kisel transistorer. Dessa metoder delas in i fyra olika delar. 1) En ny första princip metod designad för att simulera övergångsmetallföroreningar relaterade till kvantbit kandidater inom halvledare. 2) Ett teoretisk verktyg för att beräkna speciella egenskaper av kvantbitar. 3) En teoretisk modell introducerad för att beskriva initialiseringsprocessen av kvantbitar. 4) Ett abstrakt teoretisk tillvägagångssätt har används för att grundligt förstå fysiken av kvantbitar lika så specifika två-dimensionella material. 

This thesis is a result of my Ph.D. studies in the Theory and Modeling Division at Linköping University from 2012 to 2016. My results have been published in peer-reviewed journals, with the exception of Paper VI, which is under review at the Physical Review Letters journal.

Support for this research was received from Knut \& Alice Wallenberg Foundation "Isotopic Control for Ultimate Materials Properties".

The theoretical calculations have been carried out by using supercomputer resources provided by the Swedish infrastructure for computing (SNIC) at the National Supercomputer Center (NSC). 



\section{Acknowledgements}

First of all, I would like to thank my main supervisor, Prof. Igor Abrikosov, and my co-supervisors, Prof. Adam Gali and Prof. Erik Janzén, for their great guidance and helpfulness over the years. I appreciate and thank the numerous advice, the valuable discussions and the inspiring ideas. I am particularly grateful to all of them for providing and maintaining an unusual working agreement through which I can experience and learn much more than I could imagine before.

Furthermore, I am thankful to Prof. Adam Gali for launching me on my career, helping from the beginning, and for involving me in several collaborations as well as to Prof. Igor Abrikosov for showing me numerous theoretical techniques, guiding me towards method development studies, and for supporting during my PhD studies.

I would like to thank all of the members of our Swedish and Hungarian groups for creating a friendly and helpful atmosphere and for always being open for discussions.

I am also very grateful to all of my collaborators, particularly from Prof. Erik Janzén's and Prof. David Awschalom's groups and Dr. Hossein Fashandi, for the fruitful co-operations and for greatly extending my knowledge in various directions.

A doktori dolgozatom és az eddig elért eredményeim nem valósulhattak volna meg a családom folyamatos támogatása és bizalma nélkül, amelyek számos sorsfordító elhatározás meghozatalában segítettek. Szüleim és testvérem odaadása nélkülözhetetlen volt a kihívásokkal és nehézségekkel teli kutatói pályán való elinduláshoz. Szeretett feleségem pártfogása az elmúlt nyolc évben nagyban hozzájárult ahhoz hogy megfogalmazzam céljaimat és a felmerülő nehézségek ellenére is igyekezzek elérni azokat. A PhD tanulmányaim alatt rendkívüli türelmet és tartást tanúsított, valamint töretlenül támogatott és bíztatott, amelyek elengedhetetlennek bizonyultak a tanulmányaim befejezéséhez. Mindezekért öröké hálás leszek nekik! 
1 Introduction $\quad 1$

1.1 Silicon and post-silicon information processing . . . . . . . . . 2

1.2 Essential requirements and possible implementations of quantum information processing . . . . . . . . . . . . 3

1.3 Theoretical challenges in the defect spin implementation . . . . . . 3

1.4 Aim of my research . . . . . . . . . . . . . . . . . . . . 4

2 Theoretical approaches in solid state qubit studies $\mathbf{5}$

2.1 Ab initio methods . . . . . . . . . . . . . . . . . 5

2.1.1 Basic equations and approximations ........ 5

2.1.2 Density functional theory . . . . . . . . . . . . 6

2.1.3 The exchange and correlation functional . . . . . . . . 10

2.2 Model spin Hamiltonians . . . . . . . . . . . . . . . . . . . . . . . 14

2.2.1 Spin Hamiltonian of important solid state qubits . . . . . . 14

2.3 Group theory considerations . . . . . . . . . . . . . . . . . . . 17

2.3.1 Basic definitions . . . . . . . . . . . . . . 17

2.3.2 Representations................... . . 18

2.3.3 Group of the wave vector . . . . . . . . . . . . . 19

2.3.4 Character tables and product tables . . . . . . . . . . . 19

3 Hybrid-DFT $+\mathbf{V}_{w}$ scheme $\quad \mathbf{2 3}$

3.1 Analogy of generalized Kohn-Sham and quasi-particles . . . . . . . 23

3.2 Theoretical unification of hybrid-DFT and DFT $+\mathrm{U}$ methods for the treatment of localized orbitals . . . . . . . . . . . 26

3.3 Limitations of hybrid functionals in mixed systems . . . . . . . . 28

3.4 The hybrid-DFT $+\mathrm{V}_{w}$ scheme . . . . . . . . . . . . . . . . 29 
4 Zero-field-splitting tensor calculation 33

4.1 Implementation of zero-field-splitting tensor calculation . . . . . . 33

4.2 Application in qubit characterization . . . . . . . . . . . . . . . 35

4.2.1 Temperature and pressure dependence . . . . . . . . . 35

5 Model of dynamic nuclear polarization $\quad 39$

5.1 Basic properties of electron and nuclear spin hybrid registers in

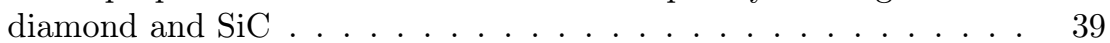

5.2 Simple model of dynamic nuclear polarization . . . . . . . . . . . . 41

5.3 Comprehensive theoretical model of DNP . . . . . . . . . . . . . 41

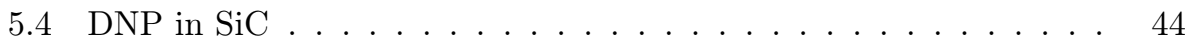

5.5 Ground state DNP of weakly coupled hybrid registers . . . . . . . 47

6 Group theory approaches $\quad 49$

6.1 Carbon antisite-vacancy pair . . . . . . . . . . . . . . 49

6.2 Dirac points in novel 2D materials . . . . . . . . . . . . 51

7 Conclusions and outlook $\mathbf{5 5}$

7.1 The hybrid-DFT $+\mathrm{V}_{w}$ scheme . . . . . . . . . . . . . . . 55

7.2 Zero-field-splitting calculation . . . . . . . . . . . . . . 56

7.3 Dynamic nuclear spin polarization .......... 56

$\begin{array}{ll}\text { Bibliography } & 57\end{array}$

List of included Publications $\quad 65$

$\begin{array}{lr}\text { Related, not included publications } & 67\end{array}$

$\begin{array}{ll}\text { Summary of included papers } & 71\end{array}$

$\begin{array}{ll}\text { Paper I } & 75\end{array}$

$\begin{array}{ll}\text { Paper II } & 91\end{array}$

$\begin{array}{ll}\text { Paper III } & 101\end{array}$

$\begin{array}{ll}\text { Paper IV } & 111\end{array}$

$\begin{array}{ll}\text { Paper V } & 131\end{array}$

$\begin{array}{ll}\text { Paper VI } & 139\end{array}$

$\begin{array}{ll}\text { Paper VII } & 159\end{array}$

$\begin{array}{ll}\text { Paper VIII } & 183\end{array}$ 


\section{CHAPTER 1}

Introduction

Information processing, i.e. gathering, handling, storing, and transferring information, played essential role in mankind's history in the last few thousand years[1]. The first important breakthrough happened presumably when people became capable of recording and transferring information to larger distances, which made governance and the formation of larger societies and nations possible. Later, the use of books for the collectivization and storage of great thinkers' knowledge established the foundations of philosophy and modern science. Advances in technology resulted in further development of information processing and society, e.g. the invention of printing greatly accelerated the development of culture and science, electric wired communication made information transfer instant, while the utilization of electromagnetic waves made broadcasting possible.

In retrospect, one can see that improvements in information processing, which were usually facilitated by newer and newer technological achievements, accelerated the social development of mankind. Alternatively, one may also say that, throughout history, technology, information processing, and society developed hand-in-hand.[1]

Unlimited and fast-achievable information is one of the ordinary resources of nowadays generations. Although, this may seem to be a well-developed stage of information processing, in science, a new revolution is unfolding in the area of post-silicon information processing technologies, which can revolutionize information processing and consequently future generations' daily life and society. The developing technologies use more advanced processing methods than the transistor effect of conventional semiconductors, as well as novel advanced materials in most of the cases. 


\subsection{Silicon and post-silicon information processing}

The beginnings of modern information communication technologies' development dates nearly seventy years back now, back to 1947, when John Bardeen, Walter Brattain, and William Shockley demonstrated the first transistor[2, 3]. Two revolutions started at that moment, the development of solid-state electronics and semiconductor technologies as well as the development of digital information processing. However, communication and computation are possible without transistors, for instance, by analog devices[4], the digitalization of the information, i.e. cutting into countless number of elementary pieces of information, such as bits, made the storage and processing much simpler[5]. Transistors, as silicon based semiconductor devices made the miniaturization of information processing electronic circuits possible[6], thus the development of processing units, containing billions of transistors, that can carry out complicated information processing operations.

From the scientist point of view, the development of transistor started much earlier in time. Several decades of research, numerous theoretical and experimental breakthroughs were needed to set up a model system, in which the basic properties of semiconductors, the effects of doping, and the nature of hetero-junctions could be understood. These theoretical models were used then to describe hypothetical devices, such as transistors, and to determine the requirements of the experimental realization.

Today's researchers may witness a similar process in science that may result in a new revolution in information processing. The new technologies under investigation utilize several achievements of silicon technologies and manufacturing, however, employ not classical but quantum dynamics for processing.

The basic idea of the potential breakthrough is simply to replace the classical bits with quantum bits (qubits) that can implement elementary quantum information. As quantum objects can have superposition states, not only two as classical bits, they can store and process multiple pieces of information parallel. Importantly, this capability increases as $2^{N}$ when $N$ two-state qubit system is considered.[7] Exploiting quantum information for computation and communication can have nearly unpredictable effect on science and the next generations' daily life. It is already known that classical secure communication channels could be broken by quantum computers [8, 9], while unbreakable quantum channels could be established[10], several algorithm could be substantially accelerated, and quantum systems could be simulated efficiently by quantum computers.[7]

The field of quantum computation was initiated by several researchers, such as Paul Benioff[11], Yuri Manin, Richard Feynman[12], and David Deutsch[13], who inspired scientists to develop quantum algorithms, such as Peter Shor's factorization algorithm[8] and Lov Grover's algorithm of quantum database search[14]. The experimental realization of qubits and qubit networks is on the other hand one of the greatest challenge in mankind's history. 


\subsection{Essential requirements and possible implemen- tations of quantum information processing}

There are several requirements, known from theoretical considerations, that every working realization of quantum computer must fulfill. Seven simple criteria were formulated by David P. DiVincenzo[15]:

1. Scalable realization of well characterized qubits

2. Possibility of initializing the system of qubits

3. Long enough coherence time to carry out quantum operations

4. Universal quantum gates

5. Quantum state read-out possibilities

6. The possibility of interconversion between stationary and flying qubits

7. Faithful transmission of flying qubits

Quantum bits can be realized in every such systems where quasi-two-level systems can be separated and manipulated individually[7]. In the past decades, numerous proposals have been presented in a wide spectrum of physical systems. Every implementation has its own advantage and disadvantage, some of them can fulfill several of the above mentioned criteria, however, so far none of the proposals could satisfy all of them. The most developed qubit implementations use, for instance, trapped ions, semiconductor quantum dots, superconducting circuits, or defect spins in solids.[16, 17]

The theoretical studies carried in the framework of this thesis are connected to the defect spin realizations of quantum bits and quantum information processing[18]. In this approach, there are several potential qubit candidates with attractive properties[19-31]. The most famous and well characterized qubits are the nitrogen-vacancy (NV) center in diamond[26], the divacancy and related centers in silicon carbide[28, 32] and the phosphor in silicon[33, 34]. All these qubits can be individually initialized and read-out by optical or electrical means, exhibit sufficiently long coherence time, and simple quantum operations have been demonstrated with them. [27, 33-40] In the first two cases, entanglement with flying qubits, such as photons, is also possible.[27, 28, 36]

Despite the enormous improvement that has been achieved during the last decades, there are still several requirements needed to be fulfilled. For instance, effectively couple qubits, without the loss of coherence, in order to realize multiqubit gate operations.

\subsection{Theoretical challenges in the defect spin imple- mentation}

As the implementation of quantum information processing is completely new direction in solid-state physics, the development of this area requires new experimental 
devices and approaches as well as new theoretical tools, models, and understanding. From the theorist point of view, there are diverse theoretical challenges, ranging from materials science issues to abstract mathematical description of quantum operations. At the present stage of the research achievements of this area, the theoretical challenges can be divided into four main categories:

\section{First principles method development for quantum bit candidate inves- tigation}

Whereas there are promising qubit candidates in different semiconductors, highly probable that there are many others with different or even better characteristics. To reveal these, both experimental and theoretical tools can be used. In the latter case, accurate but relatively cheap first principles techniques are needed, which can simulate the electronic structure as well as the optical and spin properties of individual defects embedded in a semiconducting host. At the present stage density functional theory with hybrid exchange-correlation functionals is the best alternatives, however, there are known limitations, see Chapter 3.

\section{First principles method development for quantum bit characterization}

Detailed first principles characterization of potentially interesting qubits is still not possible. Among others, multi-determinant description of the defect state, accurate determination of intra-defect interactions, or inclusion of electron-phonon coupling are of crucial importance. Two intra-defect spin-spin interaction calculations were recently implemented, such the hyperfine interaction and the spinspin contribution to the zero-field-splitting for high-spin ground state systems, see Chapter 4 for the latter.

\section{Modeling of simple quantum phenomena and operations}

In order to better understand the governing effects in simple quantum protocols, phenomenological models and new theoretical considerations are continuously needed.

Modeling of the initialization process of coupled electron spin - nuclear spin systems, or in other words hybrid registers, is discussed in Chapter 5.

\section{Developing quantum protocols and quantum algorithms}

Protocols and algorithms are already needed to accomplish simple, few qubit quantum processing. Due to the improvement of the implementations, such theoretical developments are becoming more and more indispensable.

\subsection{Aim of my research}

The aim of my research is to do relevant and valuable theoretical modeling, including method development and application, that can facilitate the development of point-defect-qubit-based solid-state quantum information processing. 


\section{CHAPTER 2}

\section{Theoretical approaches in solid state qubit studies}

As we have seen in the Introduction, there are different directions in theoretical physics in which a researcher can contribute to the development of solid-state quantum information processing. In this chapter, brief introduction to first principle electronic structure methods, model spin Hamiltonian methods, and grouptheoretical approaches are provided that will be intensively used in the subsequent chapters of this thesis.

The specific directions, in which the contributions have been achieved, are also introduced and discussed here.

\subsection{Ab initio methods}

\subsubsection{Basic equations and approximations}

Determining steady state material properties purely from the laws of quantum mechanics is an extraordinarily challenging task. The complexity of the problem exponentially increases with the number of particles. In solids, the numerous electrons and nuclei lead to a practically unsolvable many-body time independent Schrödinger equation[41]

$$
\hat{H} \Psi\left(\left\{\mathbf{r}_{i}, \sigma_{i}\right\},\left\{\mathbf{R}_{\mathbf{i}}, Z_{i}\right\}\right)=E \Psi\left(\left\{\mathbf{r}_{i}, \sigma_{i}\right\},\left\{\mathbf{R}_{\mathbf{i}}, Z_{i}\right\}\right),
$$

where $\hat{H}$ is the Hamiltonian of the system, $\Psi$ is the wavefunction of both the electrons, at coordinate $\mathbf{r}_{i}$ with spin $\sigma_{i}$, and the nuclei, at position $\mathbf{R}_{i}$ with charge $Z_{i}$.

To arrive to nowadays practice, when theoretical predictions can be quickly made with remarkable accuracy for almost all the imaginable atomic compositions and structures, nearly a century of methodological and technological development 
was needed. During these years several approximations have been suggested to Eq. (2.1) to obtain approximate and solvable models.

An important approximation, which is generally used throughout this thesis, is the Born-Oppenheimer approximation[42] assuming that the many-body problem of Eq. (2.1) is separable in the electron and nuclei degrees of freedom. As the electrons quickly adapt to the potential of the slowly moving nuclei, the electron Schrödinger equation can be solved for all the atomic arrangements separately,

$$
\hat{H}_{e}\left(\left\{\mathbf{R}_{i}, Z_{i}\right\}\right) \Phi\left(\left\{\mathbf{r}_{i}, \sigma_{i}\right\}\right)=E\left(\left\{\mathbf{R}_{i}, Z_{i}\right\}\right) \Phi\left(\left\{\mathbf{r}_{i}, \sigma_{i}\right\}\right),
$$

where $\Phi$ describes the electron wavefunction. The electron Hamiltonian,

$$
\hat{H}_{e}\left(\left\{\mathbf{R}_{i}, Z_{i}\right\}\right)=\hat{T}+\hat{U}+\hat{V}_{\text {ext }}\left(\left\{\mathbf{R}_{i}, Z_{i}\right\}\right),
$$

contains only the kinetic energy $\hat{T}$, the electron-electron interaction $\hat{U}$, and the nuclei's and the external potential $\hat{V}_{\text {ext }}\left(\left\{\mathbf{R}_{i}, Z_{i}\right\}\right)$.

An additional general approximation is the complete neglect of the atomic motions. For simplicity, the atomic structure is assumed to have $0 \mathrm{~K}$ temperature in classical sense, thus the considered configuration corresponds to a local or global minimum of the energy landscape over the atomic configuration space. This approximation is employed in the first principles calculations of the present thesis.

In periodic systems, like solids, the Bloch theorem provides a root for substantial simplification. Due to the presence of translational symmetry, a single electron wavefunction $\phi(\mathbf{r})$ can be written in the Bloch wave form[43],

$$
\phi_{k}(\mathbf{r})=e^{i \mathbf{k r}} u_{k}(\mathbf{r}),
$$

where $u_{k}(\mathbf{r})$ is a lattice periodic function and $\mathbf{k}$ is a crystal wave vector and a good quantum number of the single-particle state. Bloch's theorem transforms the problem of an infinitely large periodic system to an infinite number of smaller problems, i.e. to the determination of $u_{\mathbf{k}}(\mathbf{r})$ in the primitive cell for every possible $\mathbf{k}$ wave vectors. In practice, on the other hand, one needs only a finite set of $\mathbf{k}$ vectors to provide a suitable description of the system.

The theorem can be applied to many-particle systems, when the many-body wavefunction is expressible by the linear combination of Slater determinants of single-particle states.

Although the above mentioned approximations provide substantial simplification, the workload of the electron Schrödinger equation's direct solution still exponentially increases with the number of particles, which practically allows exact description of a handful of interacting electrons.

Further approximations can be made, for instance, in the framework of density functional theory, which provides computationally cheap, but relatively accurate approximate theories.

\subsubsection{Density functional theory}

Density functional theory (DFT) was first introduced by Thomas \& Fermi[44, 45], latter placed on a firm ground by Hohenberg \& Kohn[46], and brought into practice by Kohn \& Sham[47]. 
The ground breaking innovations of DFT are the introduction of the electron density as a main variable, which is much less complicated than the many-body wavefunction, and connecting it with important properties of the system, such as the total energy.[48]

\section{The Hohenberg-Kohn theorems}

The development of modern DFT started with the mathematically rigorous theorems of Hohenberg \& Kohn[46, 49]:

Lemma 1: For any system of interacting particles in an external potential $V_{\text {ext }}(\mathbf{r})$, the potential $V_{\text {ext }}(\mathbf{r})$ is determined uniquely, up to an additive constant, by the ground state particle density $n_{0}(\mathbf{r})$

As $V_{\text {ext }}(\mathbf{r})$ is the function of the ground state density $n_{0}(\mathbf{r})$ and $V_{\text {ext }}(\mathbf{r})$ fixes the Hamiltonian Eq. (2.3), the full many-body ground state and its properties are the functional of $n_{0}(\mathbf{r})$. Hohenberg \& Kohn's first lemma thus provides a variable transformation from the complicated wave function to the density that depends only on a single three dimensional space coordinate. This way the complexity of the original problem is embedded in unknown functionals that connect the observables with the density. The second lemma provides a principle to variationally obtain the ground state energy and density of the system:

Lemma 2: An energy functional $E_{V_{\text {ext }}}[n]$ can be defined, valid for any external potential $V_{\text {ext }}(\mathbf{r})$. The exact ground state energy of the system is the global minimum value of this functional, and the density $n(\mathbf{r})$ that minimizes the functional is the exact ground state density $n_{0}(\mathbf{r})$

The ground state energy functional for a given potential $V_{\text {ext }}(\mathbf{r})$ can be written as:

$$
E_{V_{\mathrm{ext}}}[n]=F[n]+\int V_{\mathrm{ext}}(\mathbf{r}) n(\mathbf{r}) d^{3} r
$$

where $F[n]$ is a universal energy functional that includes the kinetic energy $\hat{T}$ and the electron-electron interaction energy $\hat{U}$.

Exact solution of the many-body problem through the Hohenberg \& Kohn variational principle is only possible if the universal functional $F[n]$ is known. This functional was redefined later by Kohn \& Sham that provided a root for simple approximations.

\section{The Kohn-Sham total energy functional}

Approximations based on the use of non-interacting particles exhibit several practical advantages, e.g. the kinetic energy operator is well-defined,

$$
\hat{T}_{0}=-\sum_{i} \frac{\hbar^{2}}{2 m_{e}} \nabla_{i}^{2}
$$


as well as a set of single-particle equations are needed to be solved instead of the complicated many-body equations.

To benefit these features, Kohn \& Sham imagined an auxiliary non-interacting particle system that interact only through an effective potential, in such a way that the auxiliary system's ground state density reproduces the interacting many-body system's ground state density[47]. In this case, the Hohenberg \& Kohn universal function can be written as

$$
F[n(\mathbf{r})]=T_{0}[n(\mathbf{r})]+\frac{e^{2}}{8 \pi \varepsilon_{0}} \int \frac{n(\mathbf{r}) n\left(\mathbf{r}^{\prime}\right)}{\left|\mathbf{r}-\mathbf{r}^{\prime}\right|} d^{3} r d^{3} r^{\prime}+E_{\mathrm{xc}}[n(\mathbf{r})],
$$

where $T_{0}[n(\mathbf{r})]$ is the kinetic energy functional of the non-interacting particles, $E_{\mathrm{xc}}[n(\mathbf{r})]$ is the so-called exchange-correlation energy functional, accounting for the many-body effects of the interacting electron system, $e$ is the elementary charge, and $\varepsilon_{0}$ is the vacuum permittivity. Note, that the last term is defined by Eq. (2.7) and it includes the massive complexity of the original wave function based approach. Despite using non-interacting particles, the above transformation of the universal functional leaves the density functional theory exact, when the exact exchange and correlation functional is known.

The effective potential, sensed by the non-interacting particles, can be written as

$$
V_{\mathrm{eff}}(\mathbf{r})=V_{\mathrm{ext}}(\mathbf{r})+\frac{e^{2}}{4 \pi \varepsilon_{0}} \int \frac{n\left(\mathbf{r}^{\prime}\right)}{\left|\mathbf{r}-\mathbf{r}^{\prime}\right|} d^{3} r^{\prime}+V_{\mathrm{xc}}(\mathbf{r}),
$$

where the second term on the right hand side describes the mean-field electrostatic interaction of the non-interacting particles, while the last term accounts for the exchange-correlation effects of the physical many-body system. This potential can be defined through the functional derivative of the exchange-correlation energy functional,

$$
V_{\mathrm{xc}}(\mathbf{r})=\frac{\delta E_{\mathrm{xc}}[n(\mathbf{r})]}{\delta n(\mathbf{r})} .
$$

From the Euler-Lagrange equation of the Hohenberg \& Kohn variational problem of $N$ non-interacting particles, one can deduce that the minimizing density $n(\mathbf{r})$ can be obtained by solving the single-particle Kohn-Sham equations,

$$
\left(-\frac{\hbar^{2}}{2 m} \nabla_{i}^{2}+V_{\mathrm{eff}}(\mathbf{r})-\varepsilon_{i}\right) \varphi_{i}(\mathbf{r})=0
$$

with

$$
n(\mathbf{r})=\sum_{i}^{N}\left|\varphi_{i}^{2}(\mathbf{r})\right|
$$

Then, the ground state energy is given by,

$$
E_{V_{\mathrm{ext}}}[n]=\sum_{i} \varepsilon_{i}+E_{\mathrm{xc}}[n(\mathbf{r})]-\int V_{\mathrm{xc}}(\mathbf{r}) n(\mathbf{r}) d^{3} r-\frac{e^{2}}{8 \pi \varepsilon_{0}} \int \frac{n(\mathbf{r}) n\left(\mathbf{r}^{\prime}\right)}{\left|\mathbf{r}-\mathbf{r}^{\prime}\right|} d^{3} r d^{3} r^{\prime}
$$


However, the exact exchange-correlation functional is still unknown, the KohnSham equations provided a new base for finding simple, but sufficiently accurate approximation to the ground state density and energy of the interacting manyparticle systems.

\section{The generalized Kohn-Sham schemes}

Seidl et al. [50] showed in 1996 that the original Kohn \& Sham idea of using a noninteracting model system to rewrite the Hohenberg \& Kohn universal functional and thus to obtain self-consistent single-particle equations can be extended by including part of the electron-electron interaction.

Imagine a system of auxiliary interacting particles in such a way that part of exchange and correlation effects can be described by a single Slater determinant $\Phi$. Define an energy functional $S[\Phi]$ that accounts for the kinetic and interaction energy of the auxiliary system. For instance, the definition

$$
S[\Phi]=\left\langle\Phi\left|\hat{T}_{0}\right| \Phi\right\rangle+U[\Phi]+E_{\mathrm{sx}}[\Phi]
$$

includes a screened exact exchange energy contribution $E_{\mathrm{Sx}}[\Phi]$ beside the kinetic and static Coulomb interaction energies.

A density functional can be defined as

$$
F^{S}[n(\mathbf{r})]=\min _{\Phi \rightarrow n(\mathbf{r})} S[\Phi]
$$

By using the interacting model system's energy functional, the Hohenberg \& Kohn universal functional can be rewritten in the form

$$
F[n(\mathbf{r})]=F^{S}[n(\mathbf{r})]+R^{S}[n(\mathbf{r})]
$$

where the last term on the right hand side is defined by the equation and it is the difference of the Hohenberg \& Kohn universal functional and $F^{S}[n(\mathbf{r})]$.

The minimization of Eq. (2.5) leads to the generalized Kohn-Sham equations

$$
\left(\hat{O}^{S}+V_{\mathrm{eff}}^{R}(\mathbf{r})-\varepsilon_{i}\right) \varphi(\mathbf{r})=0
$$

where

$$
V_{\mathrm{eff}}^{R}(\mathbf{r})=V_{\mathrm{ext}}(\mathbf{r})+\frac{\delta R^{S}[n(\mathbf{r})]}{\delta n(\mathbf{r})}
$$

and $\hat{O}^{S}$ is a non-local operator in general.[50]

Although, the generalized Kohn-Sham schemes are exact theories, the residual $R^{S}[n(\mathbf{r})]$ functionals are unknown. In practice, however, the generalized KohnSham schemes lay down the theoretical framework of a new family of functionals that can include screened exact exchange like terms. 


\subsubsection{The exchange and correlation functional}

As we have seen so far, the Kohn-Sham and generalized Kohn-Sham schemes transform the original many-body wavefuntion problem to a completely different formalism that allows several approximations to be made, or in other words, approximate exchange and correlation functionals to be constructed. As the simplest proposal by Kohn \& Sham gained great popularity, it motivated several generations to follow their footsteps and develop various exchange-correlation functionals. Until today, 250-300 functionals were proposed in the literature. In this section, we consider the most important and most frequently used ones.

\section{Local and semi-local functionals}

Within the Kohn-Sham scheme only local and semi-local approximations can be made, in such a way that the effective potential $V_{\text {eff }}(\mathbf{r})$ depends only on the density and its derivatives.

Local-density approximation (LDA), proposed by Kohn \& Sham [47], is the simplest, but successful approximation for the exchange and correlation energy $E_{\mathrm{xc}}[n(\mathbf{r})]$,

$$
E_{\mathrm{xc}}^{\mathrm{LDA}}[n(\mathbf{r})]=\int e_{\mathrm{xc}}^{\mathrm{unif}}(n(\mathbf{r})) n(\mathbf{r}) d^{3} r,
$$

where $e_{\mathrm{xc}}^{\text {unif }}(n(\mathbf{r}))$ is the exchange-correlation energy per particle of a uniform electron gas of density $n(\mathbf{r})$. In atomic units

$$
e_{\mathrm{xc}}^{\mathrm{unif}}(n(\mathbf{r}))=e_{\mathrm{x}}^{\mathrm{unif}}(n(\mathbf{r}))+e_{\mathrm{c}}^{\mathrm{unif}}(n(\mathbf{r}))=-\frac{0.458}{r_{\mathrm{s}}(\mathbf{r})}-\frac{0.44}{r_{\mathrm{s}}(\mathbf{r})+7.8},
$$

where $r_{\mathrm{s}}$ is the radius of a sphere containing only a single electron. The exchange part is well-defined, while the correlation part was fitted to the exact solution of the uniform gas problem.[47]

LDA works best for slowly varying densities, which is rarely the case in reality. To construct exchange-correlation functionals that take into account the non-uniformness of the density, one can introduce dependence on the derivatives of the density. In the generalized gradient approximations (GGAs) [49, 51], any $f$ function of the derivatives can be used

$$
E_{\mathrm{xc}}^{\mathrm{GGA}}[n(\mathbf{r})]=\int f(n(\mathbf{r}), \nabla n(\mathbf{r})) d^{3} r .
$$

Theoretically or empirically motivated GGAs generally perform better than LDA, however, in solid-state applications, one particular functional gained remarkable popularity, due to its reliable and relatively accurate predictive power.[52] This functional was proposed by Perdew, Burke, and Ernzerhof [53] (PBE) as

$$
E_{\mathrm{xc}}^{\mathrm{PBE}}[n(\mathbf{r})]=\int e_{\mathrm{x}}^{\mathrm{unif}}(n(\mathbf{r})) F_{\mathrm{xc}}(n(\mathbf{r}), \nabla n(\mathbf{r})) d^{3} r
$$

where the refinement factor $F_{\text {xc }}$ was defined through the fulfillment of several known constrains of the exact exchange-correlation functional[53]. PBE functional is widespreadly used by the solid-state community. 
Though (semi)-local functionals showed unexpected predictive power and accuracy, there are well-known deficiencies, such as the absence of the derivative discontinuity of the exchange-correlation potential [54-56] and the self-interaction[57] of the Kohn-Sham particles. These failures have several manifestations, for instance, the notorious underestimation of the band gaps of semiconductors and insulators and the over delocalization of localized states.[52]

Concerning the subject of this thesis, where the band gaps of solid-state qubits' host should be reproduced, additionally, the localized defect states must be described accurately, the above mentioned shortcomings of the (semi-)local functionals turned to be critical. As nowadays computational resources allow more time-consuming and accurate calculations to be carried out, in this thesis, PBE functional is generally used to produce staring geometries and wave functions for more accurate calculations.

\section{Hybrid functionals}

Hybrid functionals were first introduced through the adiabatic connection theorem[58] by Becke[59] and later supported by rigorous mathematical framework through the generalized Kohn-Sham schemes[50].

In hybrids[60], part of the (semi-)local exchange energy is replaced by the Fock or exact exchange of the Kohn-Sham particles. For instance, in the case of the PBE0[61] hybrid functional

$$
E_{\mathrm{xc}}^{\mathrm{PBE} 0}[n(\mathbf{r}), \Phi]=\alpha E_{\mathrm{x}}^{\mathrm{ex}}[\Phi]+(1-\alpha) E_{\mathrm{x}}^{\mathrm{PBE}}[n(\mathbf{r})]+E_{\mathrm{c}}^{\mathrm{PBE}}[n(\mathbf{r})],
$$

where

$$
E_{\mathrm{x}}^{\mathrm{ex}}[\Phi]=-\frac{1}{2} \sum_{i, j}\left\langle\varphi_{i} \varphi_{j}\left|v_{e e}\right| \varphi_{j} \varphi_{i}\right\rangle,
$$

where $i$ and $j$ are quantum numbers, the summation goes over all the pairs of occupied states, and $v_{e e}$ is the bare Coulomb interaction,

$$
v_{e e}\left(\mathbf{r}, \mathbf{r}^{\prime}\right)=\frac{e^{2}}{4 \pi \varepsilon_{0}} \frac{1}{\left|\mathbf{r}-\mathbf{r}^{\prime}\right|}
$$

The mixing parameter $\alpha \leq 1$ of the PBE0 hybrid functional can be related to the static screening of the bare coulomb interaction, see Section 3.1. This parameter is set to 0.25 , which is mainly suitable for the description of $s p$ hybridized states.[61]

With the above definition of the exchange-correlation energy functional, the generalized Kohn-Sham equation of the generalized Kohn-Sham particles, see Eq. (2.16), can be written as

$$
\hat{H}_{0} \varphi_{i}(\mathbf{r})+\int V_{\mathrm{xc}}^{\mathrm{PBE} 0}\left(\mathbf{r}, \mathbf{r}^{\prime}\right) \varphi_{i}\left(\mathbf{r}^{\prime}\right) d^{3} r^{\prime}=\varepsilon_{i} \varphi_{i}(\mathbf{r}),
$$

where

$$
\hat{H}_{0}=-\frac{\hbar^{2}}{2 m} \nabla_{i}^{2}+\frac{e^{2}}{4 \pi \varepsilon_{0}} \int \frac{n\left(\mathbf{r}^{\prime}\right)}{\left|\mathbf{r}-\mathbf{r}^{\prime}\right|} d^{3} r^{\prime}+V_{\text {ext }}(\mathbf{r})
$$


The corresponding non-local exchange-correlation potential can be written in the form of

$$
V_{\mathrm{xc}}^{\mathrm{PBE} 0}\left(\mathbf{r}, \mathbf{r}^{\prime}\right)=\alpha V_{\mathrm{x}}^{\mathrm{ex}}\left(\mathbf{r}, \mathbf{r}^{\prime}\right)+\delta\left(\mathbf{r}-\mathbf{r}^{\prime}\right)\left((1-\alpha) V_{\mathrm{x}}^{\mathrm{PBE}}(\mathbf{r})+V_{\mathrm{c}}^{\mathrm{PBE}}(\mathbf{r})\right),
$$

where

$$
V_{\mathrm{x}}^{\mathrm{ex}}\left(\mathbf{r}, \mathbf{r}^{\prime}\right)=-\sum_{j} \varphi_{j}(\mathbf{r}) \varphi_{j}^{*}\left(\mathbf{r}^{\prime}\right) v_{e e}\left(\mathbf{r}-\mathbf{r}^{\prime}\right)
$$

Range separated hybrid functionals utilize distance dependent mixing of the exact exchange and the semi-local exchange functional. The HSE06 hybrid functional[62, 63] is a member of this family, with outstanding performances in semiconductors. The energy functional is defined as

$$
\begin{gathered}
E_{\mathrm{xc}}^{\mathrm{HSE} 06}[n(\mathbf{r}), \Phi]= \\
\alpha E_{\mathrm{x}}^{\mathrm{ex}, \mathrm{sr}}[\Phi]+(1-\alpha) E_{\mathrm{xc}}^{\mathrm{PBE}, \mathrm{sr}}[n(\mathbf{r})]+E_{\mathrm{x}}^{\mathrm{PBE}, \mathrm{lr}}[n(\mathbf{r})]+E_{\mathrm{c}}^{\mathrm{PBE}}[n(\mathbf{r})],
\end{gathered}
$$

while the exchange correlation potential is defined as

$$
\begin{gathered}
V_{\mathrm{xc}}^{\mathrm{HSE} 06}\left(\mathbf{r}, \mathbf{r}^{\prime}\right)= \\
\alpha V_{\mathrm{x}}^{\mathrm{ex}, \operatorname{sr}}\left(\mathbf{r}, \mathbf{r}^{\prime}\right)+\delta\left(\mathbf{r}-\mathbf{r}^{\prime}\right)\left((1-\alpha) V_{\mathrm{x}}^{\mathrm{PBE}, \mathrm{sr}}(\mathbf{r})+V_{\mathrm{x}}^{\mathrm{PBE}, \operatorname{lr}}(\mathbf{r})+V_{\mathrm{c}}^{\mathrm{PBE}}(\mathbf{r})\right),
\end{gathered}
$$

where the "sr" and "lr" superscripts represent the short and long-range part of the corresponding energy functional, respectively. The range separated functionals are defined through the range separation of the Coulomb hole and the bare Coulomb interaction kernel in the semi-local and the exact exchange part, respectively. In the latter case, a proper range separation function is used. The short-range Coulomb kernel in the HSE06 hybrid functional is defined by the error-function as

$$
v_{\mathrm{ee}}^{\mathrm{sr}}\left(\mathbf{r}-\mathbf{r}^{\prime}\right)=v_{e e}\left(\mathbf{r}-\mathbf{r}^{\prime}\right)\left(1-\operatorname{erf}\left(\mu\left|\mathbf{r}-\mathbf{r}^{\prime}\right|\right)\right) .
$$

Due to the exact exchange contribution, the resulting single-particle equations contain a non-local, orbital dependent potential term that makes the solution computationally more time-consuming. Despite the larger computational cost, by now, HSE06 functional has become a state-of-art tool in the field of solid-state physics. The success of hybrids in solid-state applications can be understood as a consequence of the reduced self-interaction error[57] and the introduction of the derivative discontinuity of the exchange-correlation potential[54-56]. These features give rise to improved band gaps and proper localizations.[64-67]

Despite the general improvement over the (semi-)local functionals, hybrid functionals can also fail due to the approximations used in the determination of the mixing and range separation parameters. For example, as the mixing parameter is a constant, the inclusion of the exact exchange of the generalized Kohn-Sham particles is space, energy, and orbital independent, which may cause inaccuracies when atoms of different characteristics are present in the system. These issues and their possible solutions are discussed in Chapter 3. 


\section{The DFT + U method}

Description of strong correlation is a notoriously difficult task in DFT. For instance, local and semi-local functionals tend to seriously underestimate the band gaps of Mott insulators, sometimes even a qualitatively wrong metalic ground state is predicted in the DFT calculations.[68-73]

In order to overcome these shortcomings, a generally applied strategy is to correct or entirely replace the energy contribution of the strongly correlated subset of the states.[68] The first such method, the LDA $+\mathrm{U}$ method, was introduced by Anisimov and co-workers[70, 74], which corrects the LDA energy functional by a Hubbard-like on-site occupation dependent term for the case of correlated $d$ and $f$-orbital related states. Later, the method was extended by applying the same correction term on other (semi-)local functionals. These methods are often called collectively as DFT $+\mathrm{U}$ methods, where "DFT" may refer to the specific functional in use.

The DFT $+\mathrm{U}$ total energy can be written as

$$
E_{\mathrm{DFT}+\mathrm{U}}[n(\mathbf{r})]=E_{\mathrm{DFT}}[n(\mathbf{r})]+E_{\mathrm{Hub}}\left[\mathbf{n}^{\sigma}\right]-E_{\mathrm{DC}}\left[\mathbf{n}^{\sigma}\right]
$$

where the on-site occupation matrix $\mathbf{n}^{\sigma}$ is defined by the correlated orbitals $\phi_{m}^{I}$ of an atom $I$ as

$$
\mathbf{n}_{m m^{\prime}}^{\sigma}=\sum_{i}\left\langle\varphi_{i} \mid \phi_{m}\right\rangle\left\langle\phi_{m^{\prime}} \mid \varphi_{i}\right\rangle
$$

and the Hubbard energy contribution is expressed as

$$
\begin{array}{r}
E_{\mathrm{Hub}}\left[\mathbf{n}^{\sigma}\right]=\frac{1}{2} \sum_{\{m\}, \sigma}\left\langle m m_{1}\left|v_{e e}\right| m^{\prime} m_{2}\right\rangle \mathbf{n}_{m m^{\prime}}^{\sigma} \mathbf{n}_{m_{1} m_{2}}^{-\sigma}+ \\
\frac{1}{2} \sum_{\{m\}, \sigma}\left(\left\langle m m_{1}\left|v_{e e}\right| m^{\prime} m_{2}\right\rangle-\left\langle m m_{1}\left|v_{e e}\right| m_{2} m^{\prime}\right\rangle\right) \mathbf{n}_{m m^{\prime}}^{\sigma} \mathbf{n}_{m_{1} m_{2}}^{\sigma} .
\end{array}
$$

For $d$ orbitals, the integrals of the bare Coulomb interaction $v_{e e}$ can be expressed by two parameters, $F_{0}$ and $J_{0} \cdot[70,72]$ The corresponding screened values, the Hubbard $U$ and the Stoner $J$ parameters, are often treated as adjustable parameters of the method.

The last term on the right hand side of Eq. (2.32) is the double counting term that eliminates the DFT energy of the correlated orbitals. As this contribution is not known explicitly, it is approximated in practice. In the most frequently used fully localized limit $[69,75]$ the double-counting term reads as

$$
E_{\mathrm{DC}}\left[\mathbf{n}^{\sigma}\right]=\frac{U}{2} n(n-1)-\frac{J}{2} \sum_{\sigma} n^{\sigma}\left(n^{\sigma}-1\right),
$$

where $n^{\sigma}=\operatorname{Tr}\left(\mathbf{n}^{\sigma}\right)$ and $n=\sum_{\sigma} n^{\sigma}$

A simplified variant of the DFT $+\mathrm{U}$ method is proposed by Dudarev et al. [71], in which $\left\langle m m_{1}\left|v_{e e}\right| m^{\prime} m_{2}\right\rangle \approx U$ and $\left\langle m m_{1}\left|v_{e e}\right| m_{2} m^{\prime}\right\rangle \approx J$ approximations are 
used. Dudarev's energy functional formulated as

$$
E_{\mathrm{DFT}+\mathrm{U}}^{\text {Dudarev }}[n(\mathbf{r})]=E_{\mathrm{DFT}}[n(\mathbf{r})]+\frac{U_{\mathrm{eff}}}{2}\left(\sum_{m, \sigma} \mathbf{n}_{m m}^{\sigma}-\sum_{m, m^{\prime}, \sigma} \mathbf{n}_{m m^{\prime}}^{\sigma} \mathbf{n}_{m^{\prime} m}^{\sigma}\right),
$$

where $U_{\text {eff }}=U-J$. In a proper basis of $\phi_{m}^{I}$ orbitals, the occupation matrix becomes diagonal and the energy functional simplifies to the form

$$
E_{\mathrm{DFT}+\mathrm{U}}^{\text {Dudarev }}[n(\mathbf{r})]=E_{\mathrm{DFT}}[n(\mathbf{r})]+\frac{U_{\text {eff }}}{2} \sum_{m, \sigma}\left(\mathbf{n}_{m}^{\sigma}-\left(\mathbf{n}_{m}^{\sigma}\right)^{2}\right) .
$$

The corresponding occupation dependent effective potential is obtained as the derivative of the total energy expression

$$
V_{\mathrm{DFT}+\mathrm{U}}^{\text {Dudarev }}[n(\mathbf{r})]=V_{\mathrm{eff}}[n(\mathbf{r})]+U_{\mathrm{eff}}\left(\frac{1}{2}-\mathbf{n}_{m}^{\sigma}\right) .
$$

The last term on the right hand side shows the effect of the DFT $+\mathrm{U}$ method on the correlated states. This term decreases and increases the energy of occupied and unoccupied correlated state, respectively, and thus opens a gap as large as $U_{\text {eff }}$, the effective Hubbard U.

The $\mathrm{DFT}+\mathrm{U}$ method turned to be very successful in describing strongly correlated insulators, however, there are known deficiencies, for instance, the nonprecisely defined double-counting term and the semi-local DFT treatment of the delocalized states.[76] There shortcoming can be partially remedied by the hybrid$\mathrm{DFT}+\mathrm{V}_{w}$ method, described in Chapter 3, which derives from the theoretical unification of hybrid-DFT and the DFT $+\mathrm{U}$ methods.

\subsection{Model spin Hamiltonians}

Model Hamiltonians are often used in physics. In the field of point-defect based solid-state quantum information processing, quantum bits are realized by the spins of paramagnetic color centers, or alternatively, by nuclear spins that are coupled to point defect spins in, so-called, hybrid register systems. To describe the processing, i.e. the time evolution, of such spins, model spin Hamiltonians are generally used in this field.[7]

In this section, important solid-state qubit implementations' model spin Hamiltonian is described.

\subsubsection{Spin Hamiltonian of important solid state qubits}

Paramagnetic point defects can be considered as the physical realization of the abstract object of a quantum bit.[16-18] Processing of such qubits is described by their spin Hamiltonian, which, in reality, always contains the fingerprints of the actual realization. Here, we consider the spin Hamoltonian of the two most successful point defect-based qubit implementations, the NV center in diamond[26] and the 
divacancy and related photo luminescence centers in silicon carbide (SiC) [28, 32], shortly the NV center and the divacancy. The features of the spin Hamiltonian must account for, for instance, the interactions with other qubits and the effects of the external means of control.

In the case of high-spin state point defects, such as the spin-1 NV center and divacancy, the spin Hamiltonian can be specified by six important terms[77],

$$
\hat{H}=\hat{H}_{\mathrm{ss}}+\hat{H}_{\mathrm{so}}+\hat{H}_{\mathrm{dd}}+\hat{H}_{\mathrm{hyp}}+\hat{H}_{\text {Zeeman }} .
$$

The first two terms on the right hand side of Eq. (2.39) describe intra-defect spin-spin and spin-orbit interactions[78], which give rise to the so-called zero-fieldsplitting of the spin states. The former can be written as

$$
\hat{H}_{\mathrm{ss}}=\hat{\mathbf{S}} \mathbf{D} \hat{\mathbf{S}}
$$

where $\hat{\mathbf{S}}$ is the electron spin operator vector and $\mathbf{D}$ is the D-tensor of the defect. In the threefold rotationally symmetric ${ }^{3} A_{2}$ ground state of the considered defects, the spin-spin interaction term simplifies to

$$
\hat{H}_{\mathrm{ss}}=D\left(\hat{S}_{z}^{2}-\frac{2}{3}\right),
$$

where $\hat{S}_{z}$ is the spin $z$-operator and $D$ is the D-parameter.

In the same symmetry, the spin-orbit interaction can be written in the form of

$$
\hat{H}_{\mathrm{so}}=\sum_{i} \lambda_{\perp, i}\left(\hat{L}_{x, i} \hat{S}_{x, i}+\hat{L}_{y, i} \hat{S}_{y, i}\right)+\lambda_{\|, i} \hat{L}_{z, i} \hat{S}_{z, i}
$$

where $\hat{S}_{k, i}$ and the $\hat{L}_{k, i}$ with $k \in\{x, y, z\}$ are the spin and orbital momentum operators of electron $i$. Due to symmetry reasons and the large energy gap of the singlet and triplet defect states, the spin-orbit contribution in the triplet ground state of the NV center and high symmetry divacancy configurations is negligible. Therefore, the zero-field-splitting is given by the spin-spin interaction term, thus $D$ is often called as the zero-field-splitting parameter. In the optically excited state, however, the spin-orbit interaction plays an important role as discussed in Section 5.1.

The last term on the right hand side of Eq. (2.39) describes the interaction with the external magnetic field, which allows direct external control of the spin state. This energy contribution can be approximately written as

$$
\hat{H}_{\text {Zeeman }}=-\frac{g_{e} \mu_{\mathrm{B}}}{\hbar} \hat{\mathbf{S}} \mathbf{B}(t),
$$

where $\mu_{\mathrm{B}}$ is the Bohr magneton, $g_{e}$ is the $g$-factor of the free electron, and $\mathbf{B}(t)$ is the external magnetic field. In general, two types of fields are used,

$$
\mathbf{B}(t)=\mathbf{B}_{\text {static }}+\mathbf{B}_{\mu \mathrm{w}}(t),
$$

where $\mathbf{B}_{\text {static }}$ is a static field that is used to split the spin states, while $\mathbf{B}_{\mu \mathrm{w}}(t)$ is an alternating magnetic field, typically of $\mu \mathrm{m}$ wavelengths, to drive spin state 
transitions. There are other non-direct means of external control that are discussed in Chapter 5.

The third and fourth terms on the right hand side of Eq. (2.39) describe the inter-defect dipole-dipole interaction and the defect spin-nuclear spin hyperfine interaction, respectively, which can be written as

$$
\begin{aligned}
\hat{H}_{\mathrm{dd}} & =\hat{\mathbf{S}} \mathbf{J} \hat{\mathbf{S}}_{2} \\
\hat{H}_{\mathrm{hyp}} & =\hat{\mathbf{S}} \mathbf{A} \hat{\mathbf{I}},
\end{aligned}
$$

where $\mathbf{J}$ and $\mathbf{A}$ are the tensors of the inter-defect and hyperfine interactions and $\hat{\mathbf{S}}_{2}$ and $\hat{\mathbf{I}}$ are the spin operator vectors of proximate defect spin and nuclear spin, respectively. Due to the usually low concentration of defects, the inter-defect distances are too large, thus $\mathbf{J}$ is too small to allow coherent coupling of two defects' spins. Therefore, inter-defect interactions are usually not included directly in the spin Hamiltonian. The concentration of the nuclear spins is, however, much larger in general, thus one may find a few nearby nuclear spins that couple relatively strongly to the considered defect's spin. Note, on the other hand, that the nuclear magneton $\mu_{\mathrm{N}}$ is three orders of magnitude smaller than the Bohr magneton $\mu_{\mathrm{B}}$ of the electron, thus the hyperfine interaction is non-negligible only at very short 1-10 $\AA$ distances. As these distances are comparable with the defect states' localization, the nuclei positions may overlap with the defect states, which gives rise to a so-called Fermi contact contribution in the $\mathbf{A}$ tensor, beside the dipole-dipole interaction of the electron and nuclear spins.[79]

In general, the hyperfine tensor $\mathbf{A}$ can be parameterized by its eigenvalues, $A_{x x}$, $A_{y y}$, and $A_{z z}$, and the direction of the third eigenvector, which can be specified by the polar and azimuthal angles $\theta$ and $\phi$, respectively. In most cases $A_{x x} \approx A_{y y}$ and therefore the $\phi$ dependence can be neglected. Hereinafter, we use the following three parameters, $A_{\|}=A_{z z}, A_{\perp}=A_{x x} \approx A_{y y}$, and $\theta$.

As dynamic nuclear polarization, which is the subject of Chapter 5 , is built on the hyperfine coupling of paramagnetic defects and nearby nuclear spins, here I explicitly specify the hyperfine Hamiltonian term in the basis of $\left|m_{s} m_{I}\right\rangle=$ $\{|0 \uparrow\rangle,|0 \downarrow\rangle,|-1 \uparrow\rangle|-1 \downarrow\rangle\}$ for the case of a spin-1 point defect and a spin- $1 / 2$ nuclear spin:

$$
\hat{H}_{\mathrm{hyp}}=\hat{\mathbf{S}}^{\mathrm{T}} \mathbf{A} \hat{\mathbf{I}}=\frac{1}{2}\left(\begin{array}{cccc}
0 & 0 & \frac{1}{\sqrt{2}} b & \frac{1}{\sqrt{2}} c_{-} \\
0 & 0 & \frac{1}{\sqrt{2}} c_{+} & -\frac{1}{\sqrt{2}} b \\
\frac{1}{\sqrt{2}} b & \frac{1}{\sqrt{2}} c_{+} & -A_{z} & -b \\
\frac{1}{\sqrt{2}} c_{-} & -\frac{1}{\sqrt{2}} b & -b & A_{z}
\end{array}\right),
$$

where

$$
\begin{array}{r}
A_{z}=A_{\|} \cos ^{2} \theta+A_{\perp} \sin ^{2} \theta \\
b=\left(A_{\|}-A_{\perp}\right) \cos \theta \sin \theta \\
c_{ \pm}=A_{\|} \sin ^{2} \theta+A_{\perp}\left(\cos ^{2} \theta \pm 1\right) .
\end{array}
$$

Due to the long-range nature of the dipole-dipole interaction, there are always numerous defect and nuclear spins that are weakly coupled to the point defect's 
spin. These couplings are not suitable for coherent manipulations while they affect the defect's spin states on an undefinable way and thus cause decoherence and the loss of spin polarization. Such effects cannot be described by the model spin Hamiltonian discussed in this section. Therefore, the Schrödinger equation of the Hamiltionan Eq. (2.39) is valid only for finite time interval, while the coherence of the spin states is preserved. This interval is defined by the coherence time $T_{2}^{\star}$, which sensitively depends on the paramagnetic defects' and nuclear spins' concentrations and thus on the quality of the sample. The effects of the decoherence can be included in the density matrix formalism.[80]

Tensors $\mathbf{D}$ and $\mathbf{A}$ and parameters $\lambda_{\perp}$ and $\lambda_{\|}$can be obtained from experimental measurements. Some of them can also be obtained by first principles calculations. Hyperfine tensor calculation is available in several ab initio codes, e.g. in the VASP[81-84]. An in-house implementation of the zero-field-splitting tensor calculation is described in Chapter 4.

\subsection{Group theory considerations}

The structure, which specifies the positions and types of the atoms, is the most important determinative property of solid-state materials, since it defines the potential and thus all the other properties, see Section 2.1.2. Due to this fundamental connection, the properties of the structure, for instance its symmetry, have also important implications on all the measurable quantities of the material. Group theory, which describes the abstract mathematics of the symmetry operations, can help us to obtain fundamental information from a system, without solving the complicated many-body problem, purely from the symmetry properties of the considered material's structure.[85]

In this section, I briefly review the basic definitions of group theory[85] in the context of the applications presented in the latter sections.

\subsubsection{Basic definitions}

Elements of a group $\mathcal{G}$ and the group operation (product $(\cdot)$ ) of the elements fulfill the following four defining conditions:

1. The group is closed under the group operation, i.e. product of two elements is also a member of the group $A B=C \in \mathcal{G}$.

2. The group operation is associative, $(A B) C=A(B C)$.

3. There exists a unit element $E$ such a way that $A E=E A=A$.

4. The group contains every elements' inverse, i.e. $E=A A^{-1}$ where $A$ and $A^{-1} \in \mathcal{G}$.

Symmetry operations, for instance rotation, reflection, and translation, of a particular point cluster $\mathcal{O}$ form a group with the group operation of composition, $(B \cdot A) \mathcal{O}=(B A) \mathcal{O}=B(A \mathcal{O})$, in other words: " $B$ acts after $A$ ". 
In this thesis, only point groups are considered. Point groups are groups of symmetry operations of such kind that keep at least one point of the space fixed. Therefore, point groups do not contain translation, but for instance rotation, reflection, and inversion.

\subsubsection{Representations}

From the quantum mechanical point of view representations and representation theory have the most important role in solid-state applications of group theory. A representation $D(\mathcal{G})$ is a group of square matrices that isomorphic or homomorphic to an abstract group $\mathcal{G}$ of symmetry operations. The elements of such matrix representations can be the matrices of the transformation of a basis, for instance, a basis of single-particle wave functions, under the symmetry operations.

The properties of symmetry group's representations are in tight connection with the properties of the electronic structure of a molecule or solid-state material of the given symmetry. Generally, the symmetry transformations, described by $D(\mathcal{G})$, leave the systems' Hamiltonian invariant, since the Hamiltonian must have the same symmetry as the atomic structure. This means that $D(\mathcal{G})$ commutes with the Hamiltonian, thus they can be brought to a diagonal or block diagonal form simultaneously.

The number of basis functions, i.e. the size of the matrices, is called as the dimension $d$ of the representation. In representation theory, it turns out that a $d$ dimensional representation can usually be expressed by the direct sum of a subset of smaller dimensional representations

$$
D(\mathcal{G})=\bigoplus_{i} a_{i} \Gamma_{i}(\mathcal{G})
$$

where the elementary representations $\Gamma_{i}(\mathcal{G})$, that cannot be expressed by other smaller dimension representations, are called as irreducible representations and $a_{i}$ gives how many times an irreducible representation is included in $D(\mathcal{G})$. A representation that can be expressed by irreducible representations is called reducible representation.

In the language of the matrices, this property means that there can be found a common unitary transformation that brings all the matrices of the $D(\mathcal{G})$ reducible representation into block diagonal form. Then, this block diagonal form shows that the basis functions of different blocks cannot mix with each other through the symmetry transformation of the basis. As the elements of $D(\mathcal{G})$ and the Hamiltonian of the system commute, the Hamiltonian can be transformed into the same block diagonal form with a proper unitary transformation. Since symmetry transformations mix the basis states inside the boxes, but leave the Hamiltonian and thus the energy eigenvalues unchanged, the basis states of a block must have the same energy. Therefore, the dimensions of the blocks of the block diagonal form of $D(\mathcal{G})$, or the dimensions of the irreducible representations of the reducible representation show the degeneracy of the states. 


\subsubsection{Group of the wave vector}

From the above discussion, one can also see that all the energy eigenstates can be associated with an irreducible representation. An important consequence of the symmetry is that the states of different irreducible representations cannot interact and mix with each other. This requirement has important implications on the band structure of solid-state materials.

In periodic systems, where Bloch's theorem is applicable, the energy levels can be assigned to $\mathbf{k}$ wave vectors, which are good quantum numbers of the states. All the possible such symmetrically non-equivalent $\mathbf{k}$-vectors can be found in the irreducible Brillouin zone. The point group operations that transform $\mathbf{k}$ into itself or into an equivalent $\mathbf{k}$-vector, form the group of the wave vector $\mathbf{k}$. States of a given $\mathbf{k}$-vector can be assigned to one of the irreducible representations of the group of the wave vector $\mathbf{k}$.

Due to symmetry requirements, bands of different representations cannot interact, thus they can cross each other. On the other hand, no band crossing is possible between bands of the same irreducible representation. When two such bands approach each other, there is an interaction between them and thus they start to mix. In this case avoided crossing can be observed in the band structure. These considerations are used to understand the existence of Dirac cones in the band structure of novel 2D materials in Section 6.2.

\subsubsection{Character tables and product tables}

The traces of the matrices of a representation are called as the characters of the representation. These $\chi_{i}$ characters are independent of the actual choice of the basis in which the matrices are given. Every irreducible representation of a given group has its own set of characters. The reducible representations' characters can be given by the characters of their irreducible representations,

$$
\chi_{D}(R)=\sum_{i} a_{i} \chi_{\Gamma_{i}}(R)
$$

where $R$ is the element of the group. Consequently, it is sufficient to specify the characters of the irreducible representations, which are usually provided in character tables. The ones belong to the $C_{1 h}$ and $C_{3 v}$ point group symmetry are given at the end of this section.

It is possible to define the direct product of irreducible representations. In the case of the direct product of two irreducible representations of the same group, the characters of the direct product representation can be given by the characters of the irreducible representations,

$$
\chi_{\Gamma_{i} \otimes \Gamma_{j}}(R)=\chi_{\Gamma_{i}}(R) \chi_{\Gamma_{i}}(R) .
$$

The direct product representation can be a reducible representation. The products of the irreducible representations of a group are usually given in multiplication tables. For the case $C_{1 h}$ and $C_{3 v}$ point groups these are given at the end of this section. 
Direct product representations are often used to determine selection rules, for instance, to find out the possibility as well as the polarization of optical transition between two states of given representations. Transition is possible if the direct product of the initial and final state's and the optical transition operator's irreducible representations contains the fully symmetric $A_{1}$ irreducible representation. The optical dipole operator transform as a vector, thus in $C_{3 v}$ and $C_{1 h}$ symmetry it transforms as the $A_{1}+E$ and $A^{\prime}+A^{\prime}+A^{\prime \prime}$ reducible representations, respectively.

\section{$C_{1 h}$ and $C_{3 v}$ character and product tables}

In this section, the character and product table of $C_{1 h}$ and $C_{3 v}$ point groups are provided. In the case of character tables, example basis functions of the representations are given in the first column.

Table 2.1. Character table of $C_{1 h}$ point group

\begin{tabular}{c|c|cc}
\hline \multicolumn{2}{c|}{$C_{1 h}$} & $E$ & $\sigma_{h}$ \\
\hline$x, y$ & $A^{\prime}$ & 1 & 1 \\
$z$ & $A^{\prime \prime}$ & 1 & -1 \\
\hline
\end{tabular}

Table 2.2. Character table of $C_{3 v}$ point group

\begin{tabular}{c|c|ccc}
\hline \multicolumn{2}{c|}{$C_{3 v}$} & $E$ & $2 \times C_{3}$ & $3 \times \sigma_{v}$ \\
\hline$z$ & $A_{1}$ & 1 & 1 & 1 \\
$R_{z}$ & $A_{2}$ & 1 & 1 & -1 \\
$(x, y),\left(R_{x}, R_{y}\right)$ & $\mathrm{E}$ & 2 & -1 & 0 \\
\hline
\end{tabular}

Table 2.3. Product table of $C_{1 h}$ point group

\begin{tabular}{c|cc}
\hline$C_{1 h}$ & $A^{\prime}$ & $A^{\prime \prime}$ \\
\hline$A^{\prime}$ & $A^{\prime}$ & $A^{\prime \prime}$ \\
$A^{\prime \prime}$ & $A^{\prime \prime}$ & $A^{\prime}$ \\
\hline
\end{tabular}


Table 2.4. Product table of $C_{3 v}$ point group

\begin{tabular}{c|ccc}
\hline$C_{3 v}$ & $A_{1}$ & $A_{2}$ & $E$ \\
\hline$A_{1}$ & $A_{1}$ & $A_{2}$ & $E$ \\
$A_{2}$ & $A_{2}$ & $A_{1}$ & $E$ \\
$E$ & $E$ & $E$ & $A_{1}+A_{2}+E$ \\
\hline
\end{tabular}




\section{CHAPTER 3}

\section{Hybrid-DFT $+V_{w}$ scheme}

Hybrid functionals have became popular in several fields of solid-state physics, thanks to their superior performance over (semi-)local exchange-correlation functionals in systems where an energy gap is present between the highest occupied and lowest unoccupied Kohn-Sham states. However, hybrids have well-known advantages, their limitations are less understood.

In this chapter, the performance of the hybrid functional approximation is investigated, particularly, for the case of correlated systems. Through the theoretical unification of the hybrid density functional theory (hybrid-DFT) and the DFT $+\mathrm{U}$ method for the treatment of localized orbitals, the static correlation effects introduced by the partial inclusion of exact exchange can be understood. Furthermore, a new method, the hybrid-DFT $+\mathrm{V}_{w}$ scheme, is developed to overcome the limitations of both hybrid-DFT and DFT $+\mathrm{U}$ methods.

\subsection{Analogy of generalized Kohn-Sham and quasi- particles}

Although hybrid functionals have firm theoretical foundations, there is no good practice for the determination of the direct interaction of the generalized KohnSham particles and the residual semi-local functional $R^{S}[n(\mathbf{r})]$. Hybrid functionals are often constructed with free parameters that are usually determined by fitting to experimental data sets.[60] Therefore, hybrids are often considered as semiempirical functionals.

On the other hand, some constructions have turned to be surprisingly powerful and robust and over-perform the existing semi-local functionals. In solid statephysics, due to the enhanced computation cost and the semi-empirical nature of the functionals, hybrids started to gain popularity only in the last decade. 
Nowadays, the HSE06 functional is generally used by the solid-state community because its superior performance.

From the definition of the HSE06 hybrid functional, given in Section 2.1.3, it is clear that only fraction of the Coulomb interaction is introduced. To understand why this can work, one can consider an analogy between the generalized KohnSham particles, which were introduced as a mathematical tool, and the quasiparticles of real physical meaning. Despite the lack of direct theoretical connection, the equations have similar form thus an analogy can be made. The quasi-particle equation can be written as

$$
\hat{H}_{0} \psi_{i}(\mathbf{r})+\int \Sigma\left(\mathbf{r}, \mathbf{r}^{\prime}, \varepsilon_{i}\right) \psi_{i}\left(\mathbf{r}^{\prime}\right) d^{3} r^{\prime}=\varepsilon_{i} \psi_{i}(\mathbf{r}),
$$

where $\psi_{i}(\mathbf{r})$ is the quasi-particle amplitude, $\hat{H}_{0}$ is the Hamiltonian of noninteracting particles, and $\Sigma\left(\mathbf{r}, \mathbf{r}^{\prime}, \varepsilon_{i}\right)$ is the non-local and energy dependent quasiparticle self-energy, which can be obtained exactly by Hedin's equations[86], but approximated in practice. In the case of hybrid functionals, the equation of the interacting generalized Kohn-Sham particles can be written in a similar form,

$$
\hat{H}_{0} \varphi_{i}(\mathbf{r})+\int V_{\mathrm{xc}}\left(\mathbf{r}, \mathbf{r}^{\prime}\right) \varphi_{i}\left(\mathbf{r}^{\prime}\right) d^{3} r^{\prime}=\varepsilon_{i} \varphi_{i}(\mathbf{r})
$$

where the non-local exchange correlation potential $V_{\mathrm{xc}}\left(\mathbf{r}, \mathbf{r}^{\prime}\right)$ can be defined, for example, by Eq. (2.30).

In the Coulomb-hole and screened-exchange (COHSEX) approximation[86], the self-energy can be written in the form of

$$
\Sigma_{\mathrm{COHSEX}}\left(\mathbf{r}, \mathbf{r}^{\prime}\right)=\Sigma_{\mathrm{SEX}}\left(\mathbf{r}, \mathbf{r}^{\prime}\right)+\Sigma_{\mathrm{COH}}\left(\mathbf{r}, \mathbf{r}^{\prime}\right)
$$

with

$$
\Sigma_{\mathrm{SEX}}\left(\mathbf{r}, \mathbf{r}^{\prime}\right)=-\sum_{j}^{\text {occ. }} \psi_{j}(\mathbf{r}) \psi_{j}^{*}\left(\mathbf{r}^{\prime}\right) W\left(\mathbf{r}, \mathbf{r}^{\prime}\right),
$$

where $W\left(\mathbf{r}, \mathbf{r}^{\prime}\right)$ describes the static screened interaction potential of the quasiparticles, and

$$
\Sigma_{\mathrm{COH}}\left(\mathbf{r}, \mathbf{r}^{\prime}\right)=\frac{1}{2} \delta\left(\mathbf{r}-\mathbf{r}^{\prime}\right) W_{p}\left(\mathbf{r}, \mathbf{r}^{\prime}\right),
$$

where $W_{p}\left(\mathbf{r}, \mathbf{r}^{\prime}\right)=W\left(\mathbf{r}, \mathbf{r}^{\prime}\right)-v_{e e}\left(\mathbf{r}-\mathbf{r}^{\prime}\right)$ and $v_{e e}\left(\mathbf{r}-\mathbf{r}^{\prime}\right)$ is the unscreened, bare Coulomb interaction potential.

The first term on the right hand side of Eq. (3.3) describes the static exchange interaction between the quasi-particles. Importantly, this terms includes a screened, non-local interaction potential $W\left(\mathbf{r}, \mathbf{r}^{\prime}\right)$. The second term describes the interaction with the Coulomb hole, which forms around the electrons due to the correlation effects. This interaction is approximated with a local potential, see Eq. (3.5). The self-energy in the COHSEX approximation shows close similarity to the hybrid functionals' mixed non-local and local exchange-correlation potential. In the PBE0 (HSE06) functional, $\alpha V_{\mathrm{x}}^{\mathrm{ex}}\left(\mathbf{r}, \mathbf{r}^{\prime}\right)\left(\alpha V_{\mathrm{x}}^{\mathrm{ex}}, \mathrm{sr}\left(\mathbf{r}, \mathbf{r}^{\prime}\right)\right)$ can be considered as an approximations to $\Sigma_{\mathrm{SEX}}\left(\mathbf{r}, \mathbf{r}^{\prime}\right)$, while the semi-local PBE exchange-correlation 
part to $\Sigma_{\mathrm{COH}}\left(\mathbf{r}, \mathbf{r}^{\prime}\right)$. Based on this analogy, the following statement can be also made: The generalized Kohn-Sham particles are approximations to the quasiparticles, therefore, the generalized Kohn-Sham eigenvalues $\varepsilon_{i}$ can be considered as rough approximations to the quasi-particle energies.[66, 67] On the other hand, as the hybrid functionals are not directly derived from the quasi-particle equation, the validity of this approximation is unknown.

In the following, I discuss my results in the context of the above described analogy and consider the mixing parameter $\alpha$ of the hybrid functionals as a screening parameter, which determines the screening of the bare Coulomb interaction.

Hybrid functionals work best when there is a gap between the highest occupied and lowest unoccupied states. In solids, these systems are the semiconductors and insulators. Generally, in such systems the quasi-particle amplitudes are less delocalized and the Coulomb interaction between the quasi-particles is not completely screened, as in metals for instance. Free-particle approximations and the use of indirect interaction through an effective (semi-)local exchange-correlation potential have turned to be less accurate in these cases. Typical errors are the underestimation of the band gaps and the over-delocalization of the single-particle states, which results in wrong charge densities. The inclusion of direct interaction of the particles through a screened Coulomb interaction increases the exchange interaction and reduces the self-interaction of the generalized Kohn-Sham particles, thus localizes the states. Furthermore, hybrids' non-local exchange-correlation potentials exhibit a discontinuous jump when the total particle number $N$ is varied around integer numbers. This discontinuity, the so-called derivative discontinuity, opens the gap between the highest occupied and lowest unoccupied states that brings the generalized Kohn-Sham eigenvalues closer to the quasi-particles energies, and thus provides accurate band gaps for semiconductors and insulators. The value of the screening parameter, $\alpha=0.25$, turned to be quite universal for conventional semiconductors and insulators. Fine tuning of the screening, however, can yield superior results.

Kohn-Sham DFT exhibits serious shortcomings in the description of strongly correlated states and materials. In the case of Mott insulators, the band gap underestimations is very crucial, in some case, a qualitatively wrong metallic ground state is predicted. The non-zero derivative discontinuity of hybrids' exchangecorrelation potential opens a gap, however, the screening parameter must be adjusted in most of the cases. The use and performance of hybrid functionals in strongly correlated materials are still debated in the literature. A theoretical explanation why and how hybrids can work in these cases is provided in Paper I, which is discussed in details in the subsequent section. 


\subsection{Theoretical unification of hybrid-DFT and $\mathrm{DFT}+\mathrm{U}$ methods for the treatment of local- ized orbitals}

As we have seen in Chapter 2, the $\mathrm{DFT}+\mathrm{U}$ method was proposed to introduce static correlation effects into (semi-)local exchange-correlation functionals, while hybrids were proposed to introduce direct interaction of the generalized KohnSham particles. In this section, it is shown by using the results of Paper I that the two methods have similar effects on localized, strongly correlated states. The provided theoretical connection allows us to better understand the performance and limitations of hybrid functionals applied on strongly correlated systems.

An important difference of the two methods is that the DFT $+\mathrm{U}$ method acts only on the subset of correlated orbitals, while hybrids are "global" in the sense that the same potential, i.e. the same equation, is used for all the generalized Kohn-Sham particles. For further discussion on this topic see Section 3.3.

To be able to make a connection, we must consider the effect of hybrid functionals only on the subset of correlated atomic-like orbitals $\phi_{m}^{I}$, where $m$ is a quantum number, usually the projection of the orbital momentum, and $I$ specifies the atomic site. Furthermore, as $\mathrm{DFT}+\mathrm{U}$ was introduced as a correction to an exchange-correlation functional, we must bring the hybrid energy functional into an appropriate form by considering it as a corrected DFT functional.

In the subsequent derivation I consider PBE0 hybrid functional, however, the steps can be easily generalized for most of the hybrid functionals. By rearranging the terms, the PBE0 exchange-correlation energy functional can be written in the form of

$$
E_{\mathrm{xc}}^{\mathrm{PBE} 0}[n(\mathbf{r}), \Phi]=E_{\mathrm{xc}}^{\mathrm{PBE}}[n(\mathbf{r})]+\alpha E_{\mathrm{x}}^{\mathrm{ex}}[\Phi]-\alpha E_{\mathrm{x}}^{\mathrm{PBE}}[n(\mathbf{r})] .
$$

The energy correction added to the semi-local PBE functional can be defined as

$$
\Delta E_{\mathrm{xc}}^{\mathrm{PBE} 0}[n(\mathbf{r}), \Phi]=E_{\mathrm{xc}}^{\mathrm{PBE} 0}[n(\mathbf{r}), \Phi]-E_{\mathrm{xc}}^{\mathrm{PBE}}[n(\mathbf{r})]=\alpha\left(E_{\mathrm{x}}^{\mathrm{ex}}[\Phi]-E_{\mathrm{x}}^{\mathrm{PBE}}[n(\mathbf{r})]\right) .
$$

The corresponding non-local potential correction can given as

$$
\Delta V_{\mathrm{xc}}^{\mathrm{PBE} 0}\left(\mathbf{r}, \mathbf{r}^{\prime}\right)=\alpha\left(V_{\mathrm{x}}^{\mathrm{ex}}\left(\mathbf{r}, \mathbf{r}^{\prime}\right)-\delta\left(\mathbf{r}-\mathbf{r}^{\prime}\right) V_{\mathrm{x}}^{\mathrm{PBE}}(\mathbf{r})\right) .
$$

Now, we consider the effect of this correction on the subset of correlated orbitals. With the definition of the on-site occupation matrix $\mathbf{n}_{m m^{\prime}}^{\sigma}$, see Eq. (2.33), the first term on the right hand side of Eq. (3.7) can be written as

$$
E_{x}^{\mathrm{ex}}\left[\mathbf{n}^{\sigma}\right]=-\frac{1}{2} \sum_{\{m\}, \sigma}\left\langle m m_{1}\left|v_{e e}\right| m_{2} m^{\prime}\right\rangle \mathbf{n}_{m m^{\prime}}^{\sigma} \mathbf{n}_{m_{1} m_{2}}^{\sigma} .
$$

The second term on the right hand side of Eq. (3.7) serves as a double counting. To determine this term, one should determine the restricted effect of the semilocal exchange-correlation functional on the subset of correlated orbitals. As this cannot be done explicitly, an approximation is employed, similarly to the DFT $+\mathrm{U}$ method. In the present derivation, the "fully localized limit" approximation[69, 
$75]$ is utilized. In contract to the DFT $+\mathrm{U}$ method, where the screened Hubbard $U$ and Stoner $J$ parameters are used, here the bare interaction strengths, $F_{0}$ and $J_{0}$, see Section 2.1.3, are used to be consistent with the restriction, in which only direct interactions of the orbitals are taken into account, thus the screening effect of the itinerant states is not considered. With these considerations, the second term on the right hand side of Eq. (3.7) can be approximated as

$$
E_{\mathrm{xc}}^{\mathrm{PBE}}\left[\mathbf{n}^{\sigma}\right]=-\frac{F^{0}-J^{0}}{2} n-\frac{J^{0}}{2} \sum_{\sigma}\left(n^{\sigma}\right)^{2} .
$$

By applying the simplifications proposed by Dudarev et al. [71], see Section 2.1.3, the correlated orbital-restricted form of Eq. (3.7) can be written as

$$
\Delta E_{x}^{\mathrm{PBE} 0}\left[\mathbf{n}_{m}^{\sigma}\right]=\frac{\alpha\left(F^{0}-J^{0}\right)}{2} \sum_{m, \sigma}\left(\mathbf{n}_{m}^{\sigma}-\left(\mathbf{n}_{m}^{\sigma}\right)^{2}\right),
$$

while the corresponding potential term writes as

$$
\Delta V_{m}^{\mathrm{PBE} 0 \mathrm{x}, \sigma}\left[\mathbf{n}_{m}^{\sigma}\right]=\alpha\left(F^{0}-J^{0}\right)\left(\frac{1}{2}-\mathbf{n}_{m}^{\sigma}\right) .
$$

The above equations show close similarities to the energy and potential correction terms of the DFT $+\mathrm{U}$ method by Dudarev et al. [71], see Eq. (2.37) and Eq. (2.38) in Section 2.1.3. From the equivalence of the form of correction, one can deduce that the two methods have the same effect on localized atomic-like orbitals. It also means that hybrids introduce a Hubbard like on-site interaction, thus they are capable of taking into account static correlation effects, as observed, for instance, in Mott insulators. This statement may not be surprising, since, according to the analogy presented in Section 3.1, hybrids' exchange-correlation functional can be considered as an approximation to the COHSEX self-energy that accounts for the static correlation effects by the screened interaction potential. Furthermore, as can be seen from the equations, the difference is only in the strength of the correction. While in the $\mathrm{DFT}+\mathrm{U}$ method, the correction strength is determined by the effective Hubbard $U$ parameter, which is usually set by hand, in hybrids the magnitude of the correction is determined by the screening parameter $\alpha$. The Hubbard $U$ defined in the hybrid functionals thus can be given as

$$
U^{\text {hybrid }}=\alpha\left(F_{0}-J_{0}\right) .
$$

Accordingly a gap of $U^{\text {hybrid }}$ is opened between the occupied and unoccupied correlated states.

From the above derived unification of the DFT $+\mathrm{U}$ method and hybrid-DFT, it is clear that hybrids can be used for correlated materials, if dynamical correlation effects are negligible. On the other hand, in this case the screening parameter of the hybrid functional must be set carefully. It is not guarantied that the standard value of 0.25 provides the appropriate Hubbard potential.

Note, on the other hand, that the correlated orbital-restriction utilized throughout the derivation of this section is a mathematical tool, introduced to understand 
the effect of hybrid functionals on the subset of correlated orbitals. It is not possible to accomplish such restriction in practice. Therefore, any change effectuated on the screening parameter will affect the treatment of all the other orbitals too. This issue is further discussed in the subsequent sections.

\subsection{Limitations of hybrid functionals in mixed sys- tems}

From the definition of the hybrid functional, one can see that the screening of the Coulomb interaction is uniform, i.e. position, energy, and orbital independent. In this section, it is shown that this approximation fails when the systems is a mixture of orbitals of different characters. The outcomes of this investigation was published in Paper II.

As we have seen in the previous section, for correlated atomic-like orbitals, the value of the Hubbard $U$ parameter is determined by the screening parameter. In such systems, where both $s p$-hybridized and correlated $d$ or $f$-like states are present, a single $\alpha$ parameter may not be sufficient to describe the two kinds of states simultaneously and accurately.

In order to investigate the performance of hybrid functionals, in particular the HSE06 functional, on mixed systems, we considered a set of experimentally wellcharacterized transitional metal impurities ( $\mathrm{Ti}, \mathrm{V}, \mathrm{Cr}, \mathrm{Fe}$, and $\mathrm{W}$ ) in convectional semiconductors, such as silicon ( $\mathrm{Si}$ ) and silicon carbide ( $\mathrm{SiC})$. The calculations were carried out in 512 and 576 atom supercells of $\mathrm{Si}$ and $\mathrm{SiC}$, respectively.

In order to measure the accuracy of the functional, we calculated the stability of the impurities' charge states with respect to the position of the Fermi-level, i.e. determined the charge transition levels according to the following formula[87]

$$
\varepsilon\left(q \mid q^{\prime}\right)=\left(E_{q^{\prime}}+E^{\mathrm{corr}}\left(q^{\prime}\right)\right)-\left(E_{q}+E^{\mathrm{corr}}(q)\right)-\varepsilon_{\mathrm{CBM}},
$$

where $E_{q}$ is the total energy and $E^{\text {corr }}(q)$ is the charge correction[88] of charge state $q$, and $\varepsilon_{\mathrm{CBM}}$ is the conduction band minimum. The charge transition levels are directly accessible in experiment and known for the considered impurities[8992].

The HSE06 functional with $\alpha=0.25$ gives a proper description of the band structures of the semiconductor hosts. Due to the uncertainty of the charge correction as well as the residual small band gap error, the estimated error of $\varepsilon\left(q \mid q^{\prime}\right)$ is about $0.1 \mathrm{eV}$.

As can be seen in Fig. 3.1, in some cases unexpectedly large errors are obtained from the comparison of the experimental and theoretical values. We identified these errors as the sign of the inaccuracies due to the incorrect Hubbard $U$ parameter at the impurity sites, which were set by the $\alpha=0.25$ screening parameter.

A possible way to overcome the limitations of the uniform screening approximation is to construct space, $\alpha=\alpha(\mathbf{r})$, or orbital dependent, $\alpha=\alpha(\Phi)$, screening parameter [93]. Accordingly, the so-called local hybrid functionals were suggested $[94,95]$. An alternative solution to this issue is discussed in the following section. 


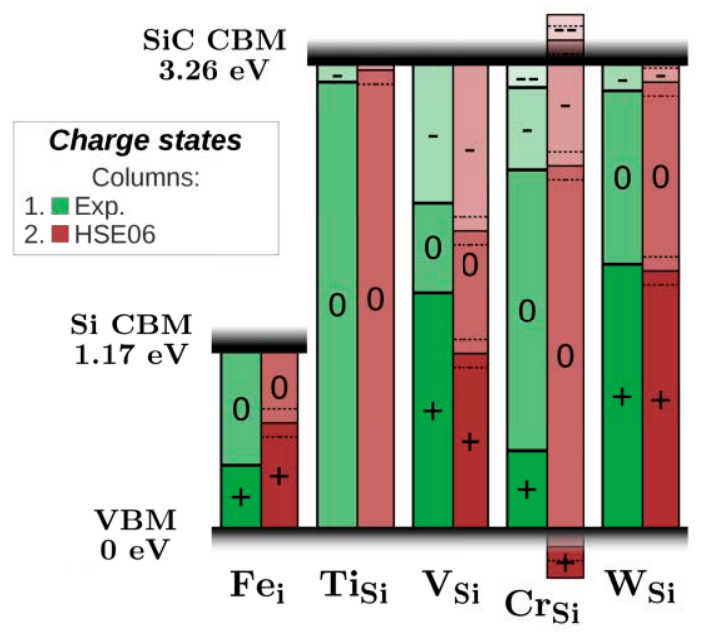

Figure 3.1. Measured and theoretically predicted charge transition levels of transition metal impurities in $4 \mathrm{H}-\mathrm{SiC}$ and $\mathrm{Si}$ hosts. In all cases the experimental results[89-92] are represented with green (first) columns where the thick horizontal line represents the charge transition levels. The results of HSE06 calculations are represented with light red (second) columns, where thin horizontal lines provide the calculated charge transition levels. The bars indicated by dashed lines around the calculated charge transition levels show the inherent uncertainty of the calculation. Constructed from the data presented in Paper II.

\subsection{The hybrid-DFT $+\mathrm{V}_{w}$ scheme}

The theoretical unification of the effect of the hybrid functionals and the DFT $+\mathrm{U}$ method on localized atomic-like orbitals allows us to construct new methods that can combine the advantage of both the hybrids and the DFT $+\mathrm{U}$ method or remedy their limitations. Such method, the hybrid-DFT $+\mathrm{V}_{w}$ scheme, was proposed in Paper I and II, which is discussed in this section.

As we have seen in the previous section, for some of the considered systems the description of the correlated orbitals is incorrect, as the global screening parameter is not suitable to provide the appropriate on-site interaction strength. Presumably this failure affects only the subset of correlated orbitals, thus an orbital dependent correction either to the screening parameter or directly to the on-site interaction potential can be a possible solution. As the form of hybrids' potential correction is identical to the one of Dudarev's implementation of the DFT $+\mathrm{U}$ method, which is available in numerous first principles codes, direct correction of the on-site interaction potential can be implemented easily.

The on-site potential that can be added to a hybrid functional's exchange and correlation potential can be written as

$$
V_{w}\left[\mathbf{n}_{m}^{\sigma}\right]=w\left(\frac{1}{2}-\mathbf{n}_{m}^{\sigma}\right)
$$




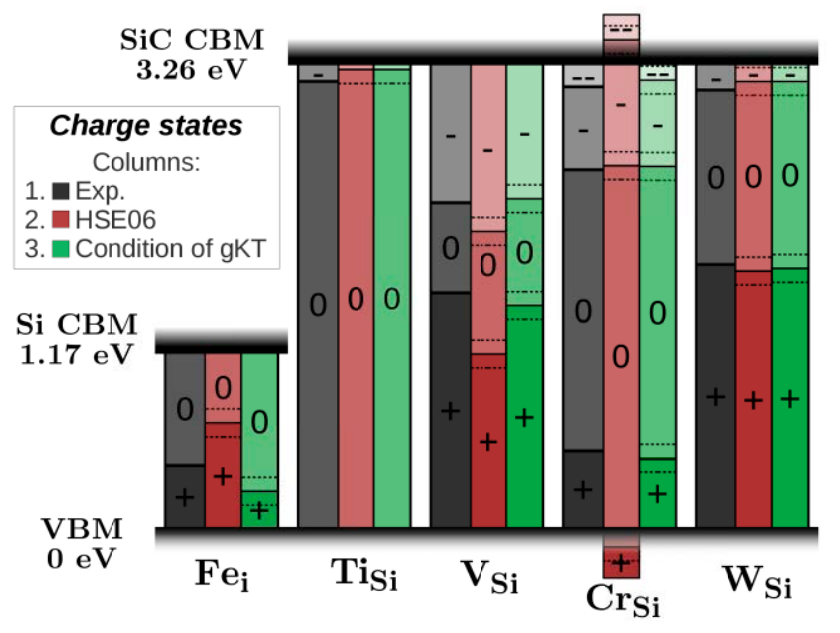

Figure 3.2. Measured and theoretically predicted charge transition levels of transition metal impurities in $4 \mathrm{H}-\mathrm{SiC}$ and $\mathrm{Si}$ hosts. The experimental results are represented with dark gray (first) columns, while the results of HSE06 and HSE06 $+\mathrm{V}_{w}$ calculations are represented with red (second) columns and light green (third) columns, respectively. The thick horizontal line represents the charge transition levels. Adopted from Paper II.

where $w$ defines the strength of the on-site potential correction. The corrected on-site screening parameter can be defined through the $w$ parameter as

$$
\alpha^{\prime}=\alpha+\frac{w}{F^{0}-J^{0}} .
$$

There are several ways how the potential correction strength $w$ can be obtained. When the real Hubbard $U$ parameter is known, $w$ can be defined trough the simple formula of

$$
w=U_{\text {real }}-\alpha\left(F^{0}-J^{0}\right) .
$$

It can also be considered as an adjustable parameter and fit it to experimental results to obtain more accurate theoretical description of the system.

Alternatively, one can determine the $w$ parameter through the fulfillment of the theoretical requirements of the exact DFT functional. One of these requirements is the so-called ionization potential (IP) [96, 97] or, in other context, the generalized Koopmans' theorem (gKT)[98, 99]. According to these theorems the highest occupied Kohn-Sham eigenvalue is equal to the negative ionization energy of the system, and furthermore it stays constant under the variation of its occupation. This requirement is usually not fulfilled by approximate exchange-correlation functionals. In such cases, when a suitable correction potential is known, by adjusting its parameters the IP and gKT can be fulfilled.

This strategy is utilized to determine the $w$ parameter and to calculate the charge transition levels of the correlated impurity systems, which were considered in the previous section. The results, obtained by the $\mathrm{HSE} 06+\mathrm{V}_{w}$ method, are 


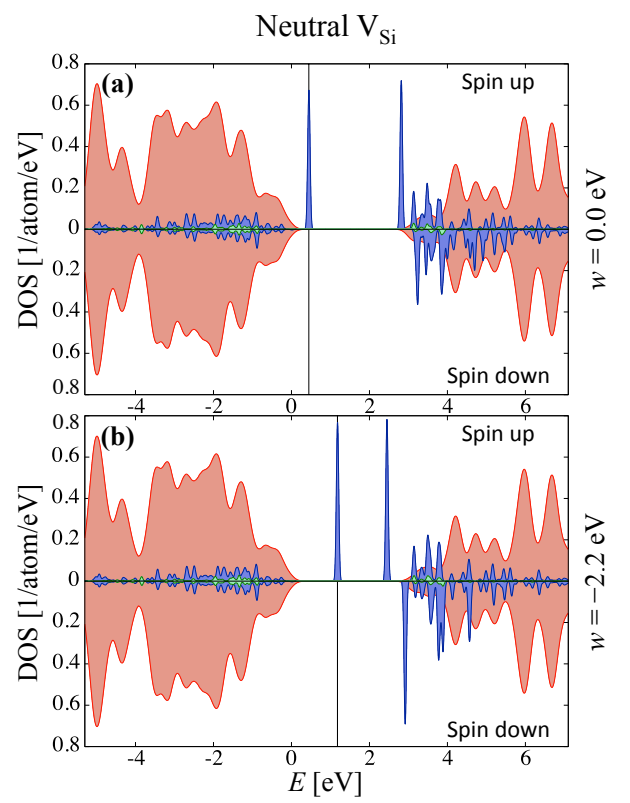

(c)

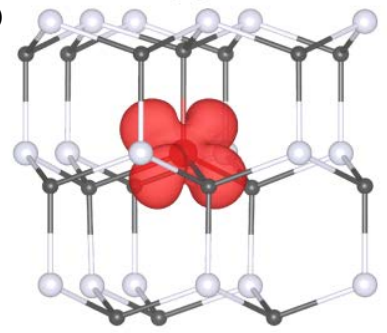

(d)

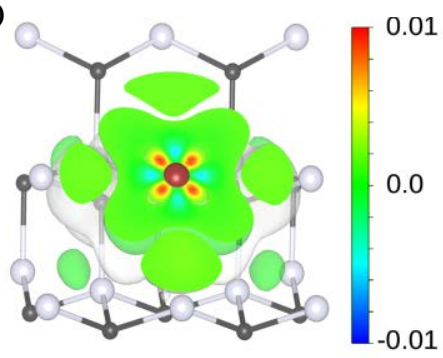

Figure 3.3. (a) - (b) Total and partial density of the states of the host 4H-SiC and the vanadium $(\mathrm{V})$ impurity in the neutral $\mathrm{V}_{\mathrm{Si}}$ point defect, respectively. The red filled curves show the total DOS of the host, while blue and green filled curves show the $d$ and $s p$ partial DOS of the vanadium. These latter curves were scaled up to be visible. Figure (a) and (b) show the results of the calculations with HSE06 and HSE06 $+\mathrm{V}_{\mathrm{w}} \mathrm{ex}^{-}$ change correlation functionals, respectively. (c) The calculated electron density (with an isosurface value of 0.05 ) of the unpaired $d$-orbital of the neutral vanadium substitutional defect in $4 \mathrm{H}-\mathrm{SiC}$. (d) $2 \mathrm{D}$ plot of the differences between two calculated charge densities using HSE06 functional and the corrected HSE06 functional around the vanadium atom. The positive finite density clearly represents the overlocalization error of the HSE06 functional. Constructed from the data presented in Paper I and Paper II.

depicted in Fig. 3.2. In all cases, when unexpectedly large errors were observed in the HSE06 calculations, the IP and gKT theorems are considerably violated. As can be seen, by applying the $V_{w}\left[\mathbf{n}_{m}^{\sigma}\right]$ on-site correction term on the HSE06 functional, and by fulfilling the requirement of the exact functional, all the charge transition levels are obtained in good agreement with the experiment.

To fulfill the theoretical requirement, negative potential corrections are needed in the case $3 d$ transitional metal impurities. This indicates that the Hubbard $U$ parameter is overestimated in the HSE06 functional. Furthermore, due to the negative correction, the corrected screening parameter is smaller than $\alpha=0.25$, which indicates that the Coulomb interaction among the impurity states is more screened than in the case of the host states. The enhanced screening effect at the impurity site can originate from the numerous unoccupied state of the transition metal elements.

The effects of the potential correction on the single-particle state can be seen 
in Fig. 3.3. Due to the negative correction, the gap between the occupied and unoccupied correlated state decreases, see Fig. 3.3(a)-(b), while the delocalization of the single-particle charge density increases, see Fig. 3.3(d). 
CHAPTER 4

\section{Zero-field-splitting tensor calculation}

In order to parameterize the model Hamiltonian of spin qubits, the inter-spin interaction strengths, for instance, the hyperfine splitting and the zero-field-splitting, are needed to be determined either by experimental measurements or by theoretical calculations. Additionally, comparison of experimental and theoretically coupling strengths may help to identify the microscopic structure of paramagnetic color centers and qubit candidates. Therefore, first principles determination of spin coupling strengths is of high importance for the development of solid-state spin-qubit-based quantum information processing.

In this chapter, the implementation of the zero-field-splitting tensor calculation and its application in the theoretical study of the pressure and temperature dependence of the NV center's zero-field-splitting parameter are discussed. Generally, there are two contributions to the zero-field-splitting of high-spin-state localized electronic states, i.e. the electron spin-electron spin dipole-dipole interaction and the spin-orbit contribution. In the considered defects in this thesis, the spin-orbit contribution is zero, due to symmetry reasons, thus hereinafter, zero-field-splitting refers only to the spin dipole-dipole interaction.

\subsection{Implementation of zero-field-splitting tensor calculation}

The implementation of zero-field-splitting tensor calculation is realized in a standalone in-house code which can use the output files of first principles plane-wave basis codes. In the current implementation, it reads the wavefunction file of the Vienna Ab initio Simulation Package (VASP)[81-83]. The details of the implementation are also discussed in Paper III. 
The $3 \times 3$ zero-field-splitting tensor $\mathbf{D}$ can be obtained from the two-particle density matrix $n\left(\mathbf{r}_{1}, \mathbf{r}_{2}\right)$, which is not accessible in DFT. On the other hand, it can be approximately determined from the two-particle density matrix,

$$
n^{(2)}\left(\mathbf{r}_{1}, \mathbf{r}_{2}\right)=\left|\Phi_{i, j}\right|^{2}=\frac{1}{2}\left|\varphi_{i}\left(\mathbf{r}_{1}\right) \varphi_{j}\left(\mathbf{r}_{2}\right)-\varphi_{i}\left(\mathbf{r}_{2}\right) \varphi_{j}\left(\mathbf{r}_{1}\right)\right|^{2},
$$

of pairs of Kohn-Sham eigenstates $\varphi_{i}(\mathbf{r})$ by using the following equation[100]

$$
D_{a b}=\frac{\mu_{0}}{4 \pi} g_{e}^{2} \beta^{2} \sum_{i<j}^{p+q} \chi_{i j} \iint n^{(2)}\left(\mathbf{r}_{1}, \mathbf{r}_{2}\right)\left(\frac{r^{2} \delta_{a b}-3 r_{a} r_{b}}{r^{5}}\right) d^{3} r_{1} d^{3} r_{2}
$$

where the summation includes every pairs of occupied Kohn-Sham states, $\chi_{i j}$ is +1 for parallel spins and -1 for antiparallel spins, $r$ and $r_{a}$ are the length and the Cartesian component of the vector $\mathbf{r}=\mathbf{r}_{2}-\mathbf{r}_{1}$, respectively, $\beta$ is the Bohr magneton, $g$ is the g-factor of the electron, and $\mu_{0}$ is the magnetic permeability of vacuum.

Note that the above equation includes every pairs of Kohn-Sham states, however, only the few highest states are spin unpaired. In DFT, the spin up and spin down Kohn-Sham states are not restricted to have the same energy and wavefunction, the states of the two spin channels are treated independently and interact through a spin dependent exchange correlation potential. This freedom allows DFT to include many-body effects, such as spin density reversal at certain atoms.

In large systems, including numerous electrons and large simulation boxes, the zero-field-splitting calculation in real space, according to Eq. (4.2), is highly inefficient compared with the momentum space expression[100],

$$
D_{a b}=\mu_{0} g_{e}^{2} \beta^{2} \Omega \sum_{i<j}^{p+q} \chi_{i j} \int n^{(2)}(\mathbf{G},-\mathbf{G})\left(\frac{G_{a} G_{b}}{G^{2}}-\frac{\delta_{a b}}{3}\right) d^{3} G,
$$

where $\mathbf{G}$ is the wave vector of the plane wave $e^{i \mathbf{G r}}$, which is used to express the single-particle wavefunctions, and $\Omega$ is the volume of the simulation box. Note that, in Eq. (4.3), only one volumetric integral must be carried out numerically.

The calculation of the two-particle density matrix, on the other hand, is efficient in real space, due to the multiplication of the Kohn-Sham single-particle wavefunctions. In the case of plane wave basis, the following strategy can be applied

$$
\begin{gathered}
f_{i j}(\mathbf{G})=\mathcal{F}\left[\mathcal{F}^{-1}\left[\varphi_{i}\right]^{*} \mathcal{F}^{-1}\left[\varphi_{j}\right]\right](\mathbf{G}) \\
n^{(2)}(\mathbf{G},-\mathbf{G})=f_{i i}(\mathbf{G}) f_{j j}(-\mathbf{G})-f_{i j}(\mathbf{G}),
\end{gathered}
$$

where $\mathcal{F}$ and $\mathcal{F}^{-1}$ represent direct and inverse Fourier transformations, respectively.

Note that the above described implementation is consistent when normconserving pseudopotential methods is used in the first principles calculations, where $\varphi_{i}(\mathbf{r})$ form an orthonormal basis. In the case of VASP, projector augmentedwave method (PAW)[101, 102] is used, in which, the PAW potentials have some 
contributions to the single-particle wavefunctions. Therefore, using normalized pseudo single-particle wavefunctions, which do not include the PAW contributions, introduces a few percent error to the calculated zero-field-splitting tensor.

As a final remark, note that the dipole-dipole interaction is a long-range interaction, goes as $1 / r^{3}$, thus in periodic boundary conditions, the spin density can interact with itself, which may cause finite-size effects in the calculated zero-fieldsplitting tensor. The investigation of this artificial self-interaction is a subject of current projects.

\subsection{Application in qubit characterization}

In a rapidly developing application of solid-state quantum bits, their wellcharacterized spin properties are used as a mean to carry out sensitive, high spatial resolution measurements. Thanks to the possibility of precise optical read-out of certain point defect qubits' spin state, small perturbations in their internal parameters, caused, for instance, by the small variation of the environment, can be measured accurately.

Along this direction, the temperature and pressure dependence of the zerofield-splitting parameter is theoretically investigated in the subsequent section.

\subsubsection{Temperature and pressure dependence}

In first principles calculations, it is usually possible to carefully investigate a system's response to an external perturbation and gain deeper insight to the phenomenon this way. The temperature and pressure dependence of the NV center's zero-field-splitting were experimentally and theoretically investigated in the literature[103-105], however, the underlying physical phenomena are still not entirely understood. Paper III contributes to this field by a first principles study, in which our in-house zero-field-splitting (ZFS) tensor calculation software is employed.

Beside the fully self-consistent ZFS calculation, several other restricted or approximate calculations can be made, in order to separate the contributions of different effects. To understand the pressure dependence of the NV center's ZFS, three models were considered. In the case of the most complete non-simplified first principles model (model 1), we applied full geometry relaxation, full self-consistent solution of the Kohn-Sham system, and a subsequent ZFS calculation. In order to separate the effects of the macroscopic compression and the microscopic structural relaxation, we ignored the latter one in our second model (model 2). To achieve this, we isotropically compressed the relaxed structure of the zero-pressure ground state and solved self-consistently the Kohn-Sham equations. Finally, to separate the effects of the change of the spatial distribution of the orbitals and the structure, in our third model (model 3) we omitted the self-consistent solution of the KS-particle system, but simulated the compression of the supercell. This can be achieved analytically by replacing the defect orbital $\varphi_{i}(\mathbf{r})$ with a spatially "scaled" and normalized one,

$$
\varphi_{i}(\mathbf{r}) \rightarrow N_{\alpha}^{-\frac{1}{2}} \varphi_{i}\left(\alpha^{-1} \mathbf{r}\right)
$$


where $\alpha$ is a scaling factor. From Eq. (4.2), one obtains that the ZFS scales as

$$
D_{\alpha}=\alpha^{-3} D_{0},
$$

where $D_{0}$ is the zero-pressure value of the ZFS and the scaling factor $\alpha$ is either chosen to be the relative change of the lattice constant $\alpha_{a}(P)=a(P) / a_{0}$ or the relative change of the carbon - carbon distance in the first neighbor shell of the carbon vacancy $\alpha_{\mathrm{C}-\mathrm{C}}(P)=d_{\mathrm{C}-\mathrm{C}}(P) / d_{\mathrm{C}-\mathrm{C}}^{0}$.

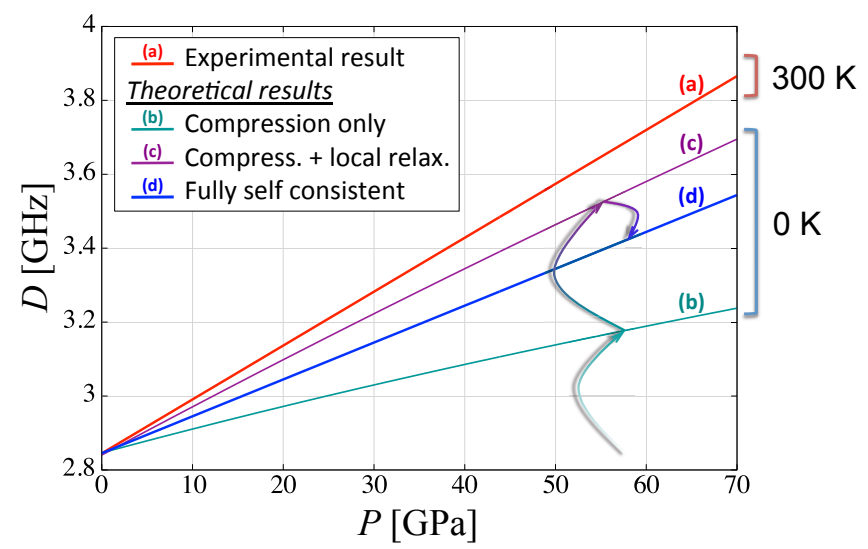

Figure 4.1. Comparison of (a) the experimental result[104] with the results of different levels of theory (b), (c), and (d). In the most simplified method (b) only the effect of the macroscopic compression of the diamond lattice is taken into account. On the next level (c) the effect of the local structural relaxation is considered, however the wave functions are kept fixed. In the fully self-consistent solution both the structure and the orbitals are relaxed. Adopted from Paper III.

In Fig. 4.1 the experimental results are plotted against different levels of theory. It can be seen from the results that the structural modification, including macroscopic compression and microscopic relaxation, has dominant contribution to the final response to the applied pressure. Note, that the fully self-consistent theoretical result still falls bellow the experimental results. The deviation can appear, for instance, due to the approximations used in the first principles calculations, i.e. due to the PBE functional, or due to the neglect of temperature effects.

The contribution of the thermal expansion to the temperature dependence of the ZFS is studied by a model, in which negative pressure is applied on the first principles model of the NV center. As can be seen in Fig. 4.2, the thermal expansion has only minor contribution to the ZFS's temperature shift. A possible explanation to the dominant effect of the temperature dependence is proposed by Doherty et al.[105]. This theory includes the effect of electron-phonon coupling. 


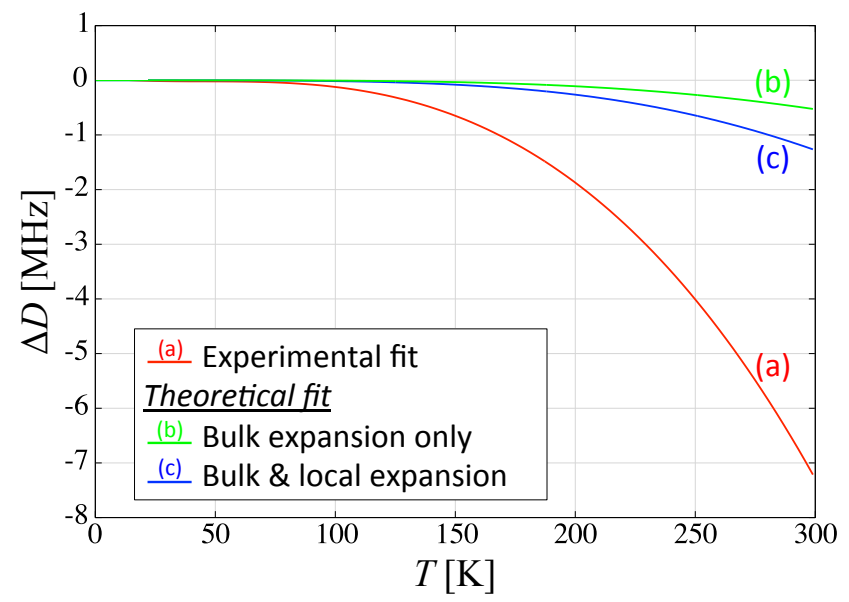

Figure 4.2. Experimental and theoretical temperature shifts of the zero-field splitting $\Delta D$ in the ground state of NV-center. Line (a) represents a fitted curve to the experimental measurements [103], while line (b) and (c) show the theoretically predicted curves in the two models. In the case of line (b) only global expansion is taken into account, while in the case of line (c) both global expansion and local structural relaxation are adopted. The orbitals are treated self consistently in both models. Adopted from Paper III. 


\section{CHAPTER 5}

\section{Model of dynamic nuclear polarization}

Nuclear spin polarization is traditionally achieved by high magnetic fields at low temperatures. Dynamic nuclear polarization (DNP), on the other hand, provides an alternative, highly efficient method for polarizing nuclear spins by transferring electron spins' polarization to the nuclei. The investigation of solid-state qubits opened a new direction for realizing novel, optically driven DNP processes. The achievable high degree of polarization in such processes makes high-fidelity nuclear spin processing and the use of hybrid register systems, in which electron spin and nuclear spin qubits are coupled, possible for quantum information processing.

In this chapter, a comprehensive theoretical model of the most important solidstate point defect qubits, the NV center in diamond and the divacancy and related centers in silicon carbide ( $\mathrm{SiC}$ ), is discussed. The model is capable of reproducing experimental results, explains the details of the phenomenon, and predicts particular effects that have been confirmed by experiment.

In the following sections, the DNP model and selected applications are discussed. Further results can be found in Paper IV, Paper V, and Paper VI.

\subsection{Basic properties of electron and nuclear spin hybrid registers in diamond and $\mathrm{SiC}$}

The most thoroughly investigated, potentially interesting paramagnetic point defect solid-state quantum bits are the NV center in diamond and the divacancy and related centers in $\mathrm{SiC}$. The two defects have similar structure, see Fig. 5.1(a), akin spin-1 electron configuration, see Fig. 5.1(b), and thus nearly the same spin Hamiltonian can be applied for both systems, see Section 2.2.

A key feature of these defects is that their spin states can be polarized by optical illumination and read-out by spin state dependent photoluminescence (PL) in 
optically detected magnetic resonance (ODMR) measurements. These mechanisms are feasible due to the possibility of spin selective non-radiative decay from the optically excited spin- ${ }^{3} E$ state, through the ${ }^{1} A_{1}$ and ${ }^{1} E$ singlet states to the spin- ${ }^{3} A_{2}$ ground state, see Fig. 5.1(c). Note, that ${ }^{1} A_{1}$ to ${ }^{1} E$ photon emission is generally not used, thus it is ignored hereinafter. The spin selectivity of the non-radiative decay is mainly due to a spin-orbit interaction mediated intersystem crossing between the ${ }^{3} E$ and the ${ }^{1} A_{1}$ states. [77, 78]

Thank to the above described electronic structure, there is a finite probability of the one-way $m_{s}= \pm 1$ to $m_{s}=0$ spin transition through the optical excitation and non-radiative decay. After a few optical cycle, the qubits are initialized in the $m_{s}=0$ state. The spin state read-out process is similarly simple, as the luminosity of the qubits depends on their spin states: In the $m_{s}= \pm 1$ state both optical and non-radiative decay are possible, while in the $m_{s}=0$ state dominantly radiative decay happens, therefore in the former case the probability of photon emission is less than that in the latter case.

(a)

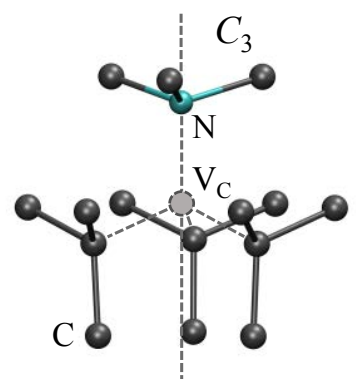

(b)

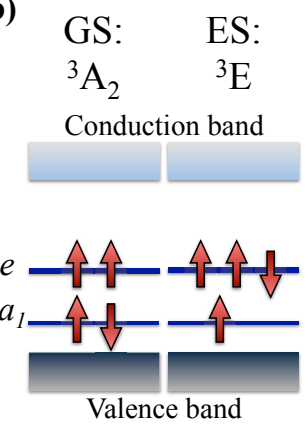

(c)

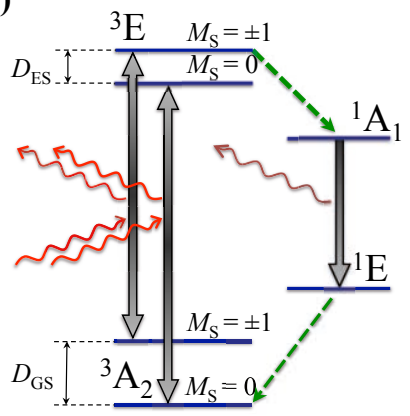

Figure 5.1. A schematic diagram of (a) the structure showing the $C_{3}$ rotation axis, (b) the electron configuration of the NV center in diamond, and (c) the NV center's optical polarization cycle. In (c), $D_{\mathrm{GS}}=2.87 \mathrm{GHz}, D_{\mathrm{ES}}=1.42 \mathrm{GHz}$ are the zero-field-splitting constants and the green dashed arrows represent the non-radiative decays, which are mediated by spin-orbit couplings and vibrations. The thick grey arrows represent the optical absorption/emission paths. The wavy lines represent photon absorption/emission in the visible (red) and near-infrared (brown) regions.

Carbon and silicon have paramagnetic isotopes in low concentrations, such as the spin- $1 / 2{ }^{13} \mathrm{C}$ and ${ }^{29} \mathrm{Si}$ with $1.1 \%$ and $4.7 \%$ natural abundance, respectively, which are randomly distributed around the NV center in diamond and divacancy in $\mathrm{SiC}$. The closer ones may couple relatively strongly to the electron spin qubit and form hybrid register systems. The NV center in diamond always forms at least a one electron - one nuclear qubit hybrid register, due to the presence of the nitrogen atom, which has only paramagnetic isotopes, such as the spin- $1{ }^{14} \mathrm{~N}$ and the spin- $1 / 2{ }^{15} \mathrm{~N}$. In the following studies, we consider only one coupled nuclear qubit, however, the results can be generalized to few nuclear qubit cases. 


\subsection{Simple model of dynamic nuclear polarization}

In the DNP process of the NV center and the divacancy, the spin polarization of the electron is transferred to nearby nuclear spin through the mixing of the electron and nuclear spin states of different spin quantum numbers. Such mixing can occur, for instance, due to the $\hat{S}_{+} \hat{I}_{-}$and $\hat{S}_{-} \hat{I}_{+}$operator combinations that appear in the hyperfine Hamiltonian term in Eq. (2.45). On the other hand, at zero magnetic field the zero-field-splitting between the $m_{s}=0$ and $m_{s}= \pm 1$ state is orders of magnitude larger than the typical hyperfine coupling strength, therefore, the mixing with the nuclear spin states is highly suppressed. Notable mixing can only occur when the hyperfine coupling is in the order, or larger than the splitting of the electron spin sublevels. By applying magnetic field parallel to the symmetry axis of the defect, the splitting of the $m_{s}=+1$ or $m_{s}=-1$ and the $m_{s}=0$ state can be brought close to the hyperfine coupling strength, see Fig. 5.2(a). When the splitting of two electron spin sublevels approaches zero, the levels do not cross due to the hyperfine coupling which opens a finite gap, see Fig. 5.2(b). This phenomenon is generally called as level anti-crossing (LAC), which can be observed both in the triplet ground state (GSLAC) and in the optically excited ${ }^{3} E$ state (ESLAC).

DNP is realized by continuous optical excitation of a hybrid register when the external magnetic field is set close to a LAC[80, 107-112]. In such circumstances, the efficiency of the $|0 \downarrow\rangle$ to $|0 \uparrow\rangle$ spin polarization process, depicted in Fig. 5.2(d), resonantly enhances.

From the application point of view, it is of high importance that what the available maximal polarization, or in other words, the fidelity of the nuclear qubit initialization is. In the steady state of the DNP process of a spin- $1 / 2$ nuclei, the nuclear polarization can be determined by the following formula, see Paper IV,

$$
P=\frac{p_{+}-p_{-}}{p_{+}+p_{-}+\kappa}
$$

where $p_{+}$and $p_{-}$are the rates of nuclear spin raising and lowering, respectively, and $\kappa$ is the rate of nuclear spin flip-flops caused by the environment. In the previous model by Jacques et al. [109], these rates were determined from the mixing of the states as

$$
p_{+}(B)=p_{-}(-B)=2|\alpha(B)|^{2}|\beta(B)|^{2},
$$

where $\alpha$ and $\beta$ are defined by the mixed eigenstate $|+\rangle=\alpha|0 \downarrow\rangle+\beta|-1 \uparrow\rangle$ of the spin Hamiltonian $\hat{H}$. In this model, $P$ can be determined as a function of the parameters of the spin Hamiltonian, for instance the strength of the external magnetic field $B$.

\subsection{Comprehensive theoretical model of DNP}

The definition of the nuclear spin flip-flop rates of the previous model is valid only in the simplest cases, when the nuclear spin is located on the symmetry axis of the defect. In such circumstances, mixing can only occur due to the $\hat{S}_{ \pm} \hat{I}_{\mp}$ operator 

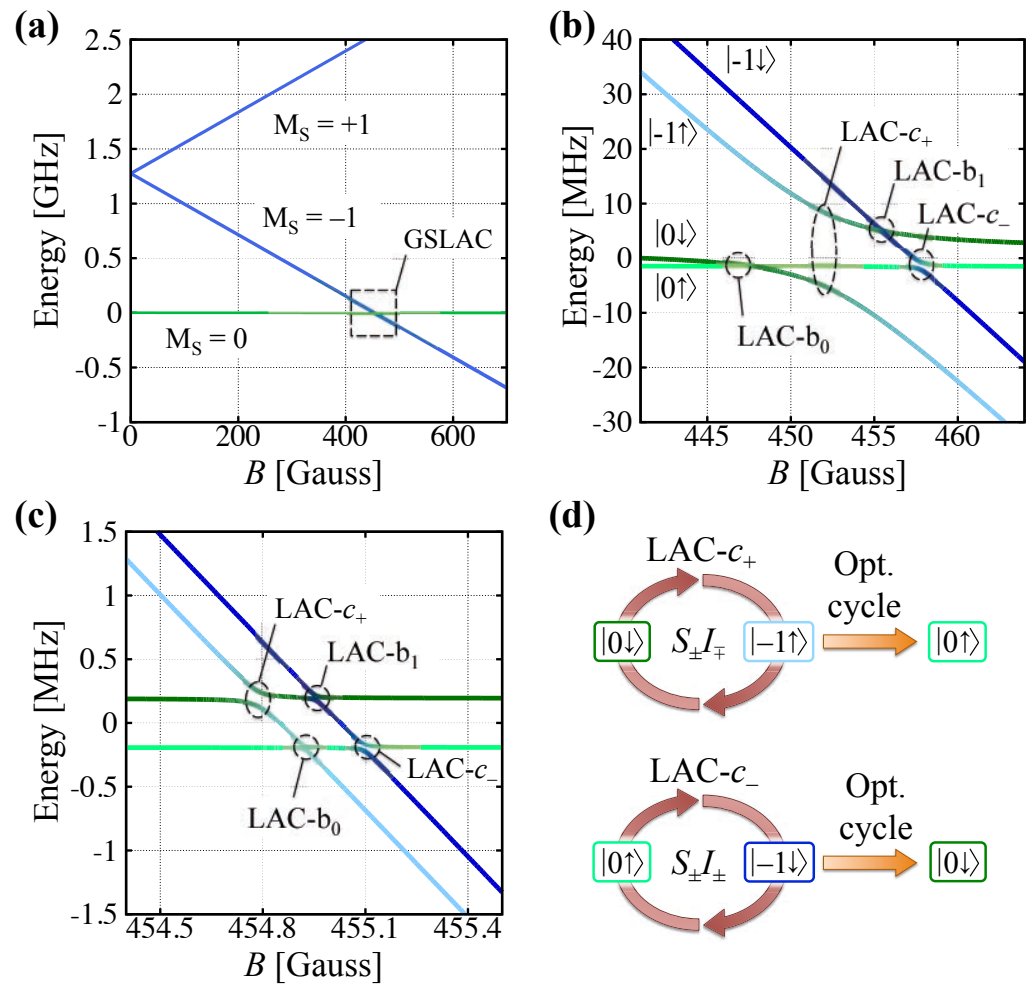

(d)
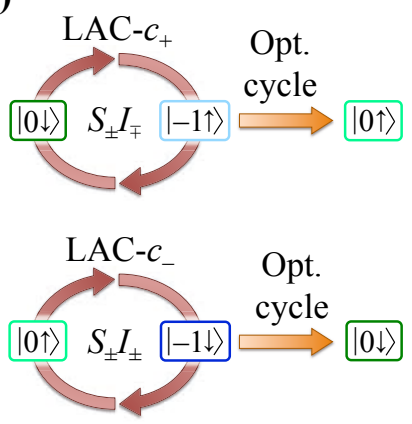

Figure 5.2. Magnetic field dependence of the ground state fine and hyperfine structure of the $6 \mathrm{H}-\mathrm{SiC} h h$ divacancy and an adjacent ${ }^{29} \mathrm{Si}$ nucleus. (a) Spin sublevels as a function of an axially applied magnetic field. Due to the zero-field splitting and the Zeeman effect, the $m_{s}=-1$ and $m_{s}=0$ spin sublevels cross at $B_{\mathrm{GSLAC}}$. (b) Magnified view of the GSLAC region for the case of ${ }^{29} \mathrm{Si}$ nucleus at the $\mathrm{Si}_{\text {IIb }}$ site[106]. Due to the low symmetry of the hyperfine field at this site, four level-anticrossings form. (c) The case of a weakly coupled nucleus, when the nuclear Zeeman splitting determines the structure of the GSLAC region. $A_{\perp} \sim A_{\|} \sim 0.1 \mathrm{MHz}$. (d) Schematic diagrams of the polarization processes at LAC-c + and LAC-c - that result in positive (upper chart) and negative (lower chart) nuclear spin polarization, respectively. In figure (a)-(c), the colors of the energy levels indicate the corresponding spin states: Blue and green lines represent $m_{s}= \pm 1$ and $m_{s}=0$ electron spin states, while lighter and darker shades of the colors represent nuclear spin up and down projections, respectively. At the LACs, the mixing of the energy levels' colors represents the mixing of the spin states.

combinations, which mix the $|0 \downarrow\rangle$ and $|-1 \uparrow\rangle$ states. This model can describe, for instance, the DNP of ${ }^{15} N$ of the NV center in diamond.

In the general case, however, the nuclear spin of the hybrid register is located out of the symmetry axis of the defect, thus the symmetry of the spin Hamiltonian is reduced. As a consequence, more complex mixing can occur due to the several nonzero matrix elements in the spin Hamiltonian, see Eq. (2.47). The model proposed in Paper IV is capable of handling general hyperfine couplings, furthermore, 
includes interactions both in the excited and ground states simultaneously, and takes into account the important effect of the electron spin depolarization due to the environment.

The key quantities in the description of the steady state nuclear spin polarization are the flipping rates or probabilities, which are determined according to the followings: As hyperfine mixing can happen between both the $m_{s}=\{0,-1\}$ and $m_{s}=\{0,+1\}$ states, with resonances at the vicinity of $\pm B_{\mathrm{LAC}}$, respectively, the total nuclear spin flipping probabilities, $p_{+}$and $p_{-}$, can be divided up into two parts,

$$
\begin{aligned}
& p_{+}=p_{+}^{(-1)}+p_{+}^{(+1)}, \\
& p_{-}=p_{-}^{(-1)}+p_{-}^{(+1)},
\end{aligned}
$$

where $p_{+}^{(-1)}$ and $p_{-}^{(-1)}$ and $p_{+}^{(+1)}$ and $p_{-}^{(+1)}$ represent nuclear spin raising and lowering rates due to the mixing in the subspace of $m_{\mathrm{s}}=\{0,-1\}$ and $m_{\mathrm{s}}=\{0,+1\}$, respectively. Note that only $p_{ \pm}^{(-1)}\left(p_{ \pm}^{(+1)}\right)$ enhances resonantly when the magnetic field approaches the LAC at $+B_{\mathrm{LAC}}\left(-B_{\mathrm{LAC}}\right)$. Considering the $m_{s}=\{0,-1\}$ branch and $B_{\mathrm{LAC}}>0$, the flipping probabilities per optical cycle can be written as

$$
\begin{aligned}
& p_{+}^{(-1)}=\sum_{\chi_{i}} p^{\mathrm{GS}}\left(0 \downarrow \mid \chi_{i}\right)\left[p^{\mathrm{ES}}\left(\chi_{i} \mid-1 \uparrow\right) \Gamma+p^{\mathrm{ES}}\left(\chi_{i} \mid 0 \uparrow\right)\right], \\
& p_{-}^{(-1)}=\sum_{\chi_{i}} p^{\mathrm{GS}}\left(0 \uparrow \mid \chi_{i}\right)\left[p^{\mathrm{ES}}\left(\chi_{i} \mid-1 \downarrow\right) \Gamma+p^{\mathrm{ES}}\left(\chi_{i} \mid 0 \downarrow\right)\right],
\end{aligned}
$$

where $\Gamma$ is the probability of non-radiative decay and $p^{\mathrm{St}}\left(\chi_{\text {Initial }} \mid \chi_{\text {Final }}\right)$ is the probability of the ground and excited state spin flip-flops, where $\chi_{\text {Initial }}$ and $\chi_{\text {Final }}$ represent the initial and final spin configurations, respectively. The above expressions sum the probabilities of all the possible spin rotation processes in the $m_{s}=\{0,-1\}$ branch that flip the nuclear spin within an optical excitation cycle. Accordingly, the summation goes over all the possible intermediate states: $|0 \uparrow\rangle,|0 \downarrow\rangle,|-1 \uparrow\rangle$, and $|-1 \downarrow\rangle$. The probabilities $p^{\mathrm{GS}}\left(\chi_{\text {Initial }} \mid \chi_{\text {Final }}\right)$ for the ground state are calculated through the time averaging of $\left|\left\langle\chi_{\text {Final }} \mid \chi(t)\right\rangle\right|^{2}$ as

$$
p^{\mathrm{GS}}\left(\chi_{\text {Initial }} \mid \chi_{\text {Final }}\right)=\sigma_{\mathrm{I}, \mathrm{F}}^{\mathrm{GS}} \frac{1}{T_{\mathrm{GS}}} \int_{0}^{T_{\mathrm{GS}}}\left|\left\langle\chi_{\text {Final }}\left|e^{-i \hat{H}^{\mathrm{GS}} t / \hbar}\right| \chi_{\text {Initial }}\right\rangle\right|^{2} d t,
$$

where the integration time $T_{\mathrm{GS}} \rightarrow \infty$, which indicates that the ground state time evolution is long enough to the oscillatory probabilities average out completely. The factor $\sigma_{\mathrm{I}, \mathrm{F}}^{\mathrm{GS}}$ takes 1 when the initial and final states are the same and takes $0<\mu<1$ when spin rotation occur. $\sigma_{\mathrm{I}, \mathrm{F}}^{\mathrm{GS}}$ and parameter $\mu$ are introduced to account for the reduction of the correlation between certain spin states, due to random spin flip-flops caused by the environment. $\mu$ is a free parameter of our model that relates to the ground state electron spin relaxation time.

The lifetime and spin relaxation time are short in the excited state, therefore there is no sufficient time for the spin rotation processes to average out. The flipping probabilities, correspond to the excited state's time evolution thus calculated 
as

$$
p^{\mathrm{ES}}\left(\chi_{\text {Initial }} \mid \chi_{\text {Final }}\right)=\int_{0}^{T_{\mathrm{ES}}} \varrho(t)\left|\left\langle\chi_{\text {Final }}\left|e^{-i \hat{H}^{\mathrm{ES}} t / \hbar}\right| \chi_{\text {Initial }}\right\rangle\right|^{2} d t,
$$

where $\varrho(t)$ is the probability distribution function of the effective length of the excited state's evolution time. $\varrho(t)$ is assumed to be an exponential distribution

$$
\varrho(t)=\frac{1}{\tau_{\mathrm{ES}}^{*}} e^{-t / \tau_{\mathrm{ES}}^{*}},
$$

where $\tau_{\mathrm{ES}}^{*}$ is the characteristic time of the decay. In our model, $\tau_{\mathrm{ES}}^{*}$ is the average effective time of evolution in the excited state, which is considered to be proportional to the excited state's lifetime $\tau_{\mathrm{ES}}$,

$$
\tau_{\mathrm{ES}}^{*}=\nu \tau_{\mathrm{ES}},
$$

where $0<\nu<-1$ is an other free parameter of the model that describes the effect of electron spin relaxation in the excited state.

This model has three parameters, $\mu, \nu$, and $\kappa$, that are treated as free parameters. All of them are related to electron or nuclear spin relaxation effects.
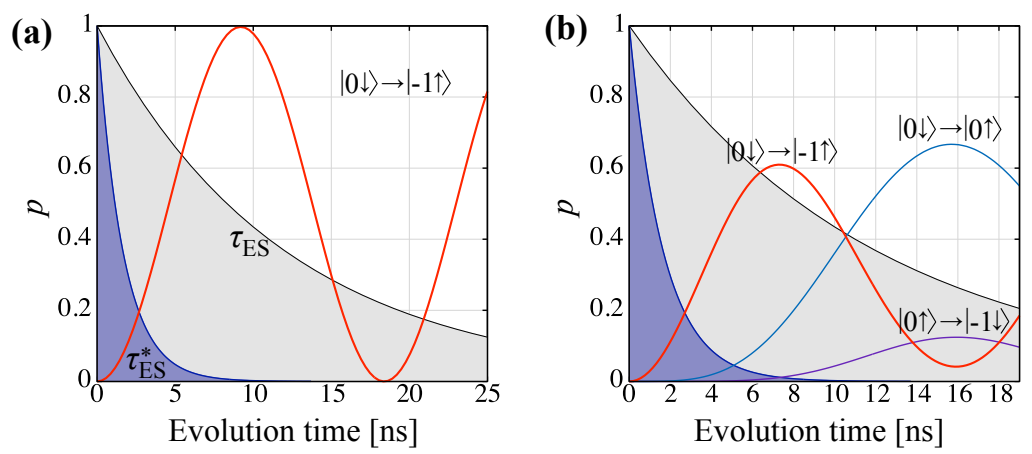

Figure 5.3. The time dependence of spin flipping probabilities in the excited state of the NV center in diamond with a ${ }^{15} \mathrm{~N}$ nucleus. (a) The magnetic field is well aligned with the axis of the defect. In this case, only one spin rotation can occur, $|0 \downarrow\rangle \rightarrow|-1 \uparrow\rangle$, represented with red (thick gray) line. The black and blue lines with light gray and blue filled areas represent the exponential decay of the excited state lifetime and the effective evolution time, respectively. The mean evolution time can be seen to be much shorter than the periodicity of the oscillatory probability. (b) The magnetic field is misaligned by $1^{\circ}$. In this case, different kinds of spin rotations occur that lower the polarizability of the nuclear spin (Not all processes are depicted). Adopted from Paper IV.

\subsection{DNP in $\mathrm{SiC}$}

Divacancy and related photo luminescence centers' DNP is first investigated in Paper $\mathrm{V}$, where remarkably high, $99 \pm 1 \%$ nuclear spin polarization is reported in the 
ESLAC region. These results make SiC potentially interesting for the investigation of hybrid registers.

Interestingly, not all the divacancy related centers show outstandingly efficient DNP. As the parameters of the spin Hamiltonian, such as the hyperfine coupling strength, are rather similar for all the configurations, the observed large deviations in the maximal nuclear spin polarization are surprising. This phenomenon is investigated and understood by the extended DNP model introduced in Paper IV.

In the case of different divacancy qubits in $\mathrm{SiC}$, the excited state lifetime is nearly the same[113], however, the spin dephasing time varies substantially, which may indicate different spin relaxation times. In our DNP model, such effects are included through a reduced, effective lifetime $\tau_{\mathrm{ES}}^{*}=\nu \tau_{\mathrm{ES}}$ in the excited state.

The reason behind the different DNP efficiency is the short and varying effective lifetime in the excited state. The reasons can be understood with the help of Fig. 5.3. When the timescale of the spins' free evolution is shorter than the periodicity of the spin rotation processes, the probability of the nuclear spin flip-flops is highly reduced, as there is no sufficient time for the mixing of the states. In such circumstances the flipping probabilities sensitively depend on the actual value of the effective lifetime.

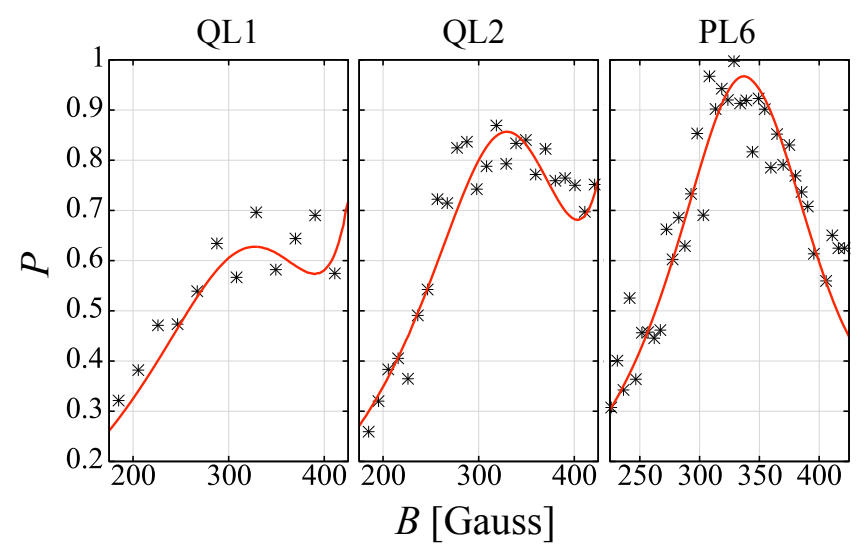

Figure 5.4. The adjusted theoretical curves (solid lines) and the experimental measurements (points) on the dynamic nuclear spin polarization of different divacancy configurations and related defects in SiC. The panels show the QL1, QL2 and PL6 photoluminescence centers (ordered from left to right). The first two centers are assigned to the $k_{1} k_{1}$ and $h h$ configurations of divacancy in $6 \mathrm{H}-\mathrm{SiC}$. The unidentified PL6 center is modeled by hyperfine parameters of $k_{2} k_{2}$ configuration in the theoretical simulations. Adopted from Paper IV.

In order to be able to reproduce the experimental observations, see Fig. 5.4, one has to adjust the $\nu$ parameter, which is related to the excited state spin relaxation processes. The fitted effective time constants, $\tau_{\mathrm{ES}}^{*}=\nu \tau_{\mathrm{ES}}$, turned to be in-line with the trends of the reported decoherence time constants.

The GSLAC DNP process of divacancy and related qubits was first theoretically and experimentally investigated in Paper VI. Due to the longer evolution time as 
(a)

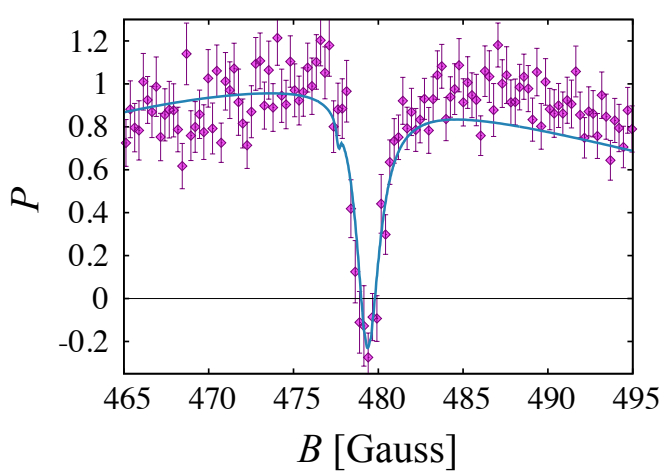

(b)

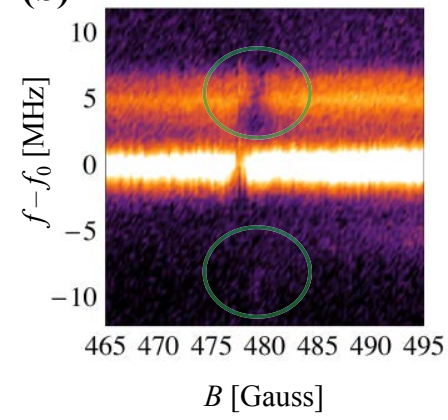

(c)

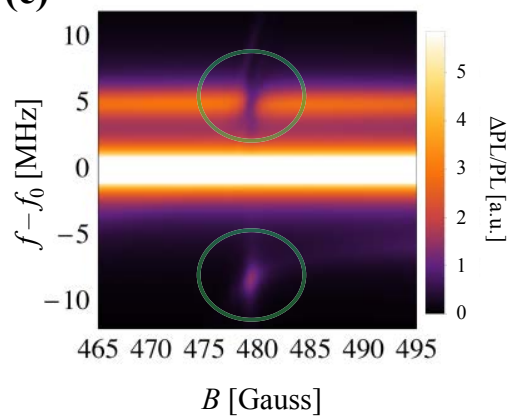

Figure 5.5. Experimental and theoretical ${ }^{29} \mathrm{Si}$ nuclear spin polarization and ODMR spectrum of the $m_{s}=0$ to $m_{s}=+1$ spin transition of PL6 qubits in $4 \mathrm{H}-\mathrm{SiC}$ at the GSLAC region. (a) The measured (points) and calculated (thick line) magnetic field dependence of the nuclear spin polarization of a ${ }^{29} \mathrm{Si}$ nucleus at the SiIIb site. DNP is highly efficient up until the $\mathrm{LAC}-c_{-}$, at which point it exhibits a sharp drop and reversal. Measurements are carried out at room temperature. (b) The experimental lowmicrowave-power ODMR spectrum. The measurements are carried out on an ensemble of PL6 divacancy relate qubits in $4 \mathrm{H}-\mathrm{SiC}$ at room temperature. (c) Theoretical simulation of the ODMR spectrum, takes into account the DNP of the ${ }^{29} \mathrm{Si}$ nucleus at the $\mathrm{Si}_{\mathrm{IIb}}$ site and the microwave transition strength in the $m_{s}=\{0,+1\}$ manifold. The green ellipsoids in (b) and (c) highlight the indications of the nuclear spin polarization reversal. Constructed from the data presented in Paper VI.

well as the low symmetry of the hyperfine field, complex DNP features can be observed.

In low symmetry, not only $\hat{S}_{ \pm} \hat{I}_{\mp}$ but for instance $\hat{S}_{ \pm} \hat{I}_{ \pm}$operator combinations appear in the spin Hamiltonian, see Eq. (2.47). In the excited state, due to the efficient suppression of the slow spin rotation process, only the strongest hyperfine coupling term can have an effect. In the ground state, on the other hand, weaker hyperfine coupling terms, i.e. slower processes, can contribute to the DNP process. Consequently, the process governed by $\hat{S}_{ \pm} \hat{I}_{ \pm}$operators, which polarizes the nuclear spin in the $|\downarrow\rangle$ spin state, may cause a polarization drop or inversion in the $P(B)$ 
curve. Additionally, this process causes a narrow LAC at the crossing of the $|0 \uparrow\rangle$ and $|-1 \downarrow\rangle$ states, see Fig. 5.2(b).

The calculations predicted a polarization inversion in GSLAC DNP of the divacancy qubits, which was experimentally confirmed, see Fig. 5.5.

\subsection{Ground state DNP of weakly coupled hybrid registers}

At the end of the previous section, the GSLAC DNP of nearby nuclear spin was theoretically and experimentally investigated. Particularly, nuclear spins at the $\mathrm{Si}_{\text {IIb }}$ site[106] of the divacancies, see Paper V, were considered, where the strength of the hyperfine interaction is approximately $10 \mathrm{MHz}$. In this case the details of the polarization inversion is determined dominantly by the hyperfine interaction. Unfortunately, the LACs, correspond to the positive and negative polarization processes, are too wide and too close to each other, thus they overlap. As a consequence, the up and down polarization processes mutually reduces the efficiency of the other process.

In Paper VI, the GSLAC DNP of weakly coupled nuclei is studied. For sufficiently small values of the hyperfine coupling strength, $|A|<0.4 \mathrm{MHz}$, the nuclear Zeeman splitting becomes the dominant interaction at the LAC region, and thus it determines the positions of the crossings of the energy levels, see Fig. 5.2(c). Therefore, even if the hyperfine splitting is small, there still exists a finite magnetic field difference between the different LACs. In our simulations, this difference turned to be sufficient to minimize the overlap of the competing processes, thus their efficiency can be maximal.
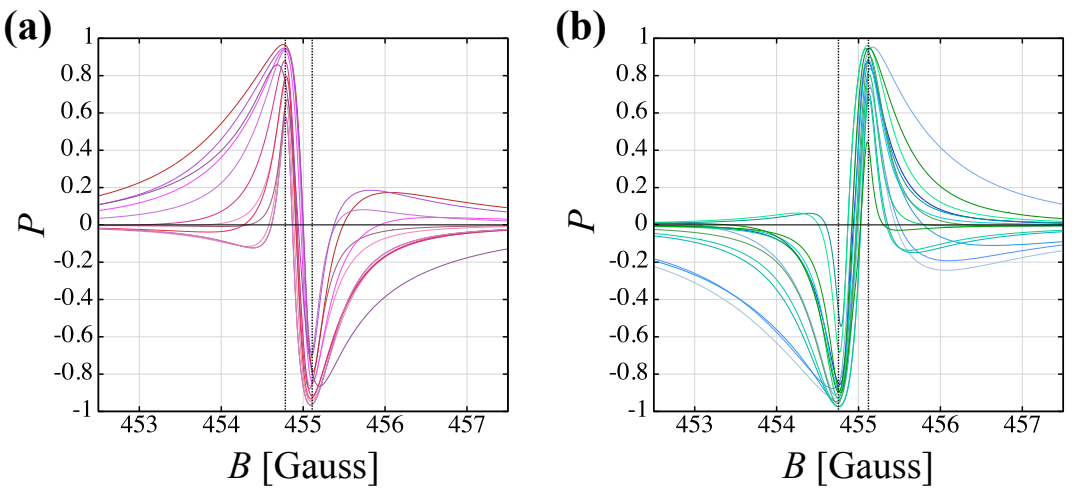

Figure 5.6. Magnetic field dependence of the DNP of (a) ${ }^{29} \mathrm{Si}$ and (b) ${ }^{13} \mathrm{C}$ nuclei at different weakly coupled neighboring sites around the $h h$ divacancy in $6 \mathrm{H}-\mathrm{SiC}$. Most of the polarization curves on (a) and (b) show "peak and dip" structure with a strong magnetic field dependence. Due to the opposite sign of the nuclear $g$-factor of ${ }^{29} \mathrm{Si}$ and ${ }^{13} \mathrm{C}$, the order of the peak and the dip is opposite for the two nuclei. Constructed from the data presented in Paper VI. 
The simulated $P(B)$ polarization curves show a well developed "peak and dip" structure, see Fig. 5.6, demonstrating the separation of the spin polarization process. Note that the polarization inversion is nearly from $100 \%$ to $-100 \%$. Considering all the possible nuclei sites in a sphere of $10 \AA$ around a divacancy qubit, we find this DNP feature in nearly $50 \%$ of the cases, which shows the generality of the prediction among the weakly coupled hybrid registers.

As nuclear spin polarization can be controlled by magnetic field variations as small as 0.3 Gauss, our finding offers new avenues for radio-frequency-free, magnetic-field-controlled high fidelity nuclear qubit initialization. Among other possibilities, qubit state dependent nuclear qubit initialization could be realized with a pair of neighbor hybrid registers. 


\section{CHAPTER 6}

\section{Group theory approaches}

Group theory in solid-state physics is an already well-developed abstract theoretical framework, which can be very powerful, especially when it is combined with first principles calculations. In this chapter, two examples are provided where joined first principles and group theoretical analysis is employed.

In the first example, group theory is utilized to analyze the configuration, electronic structure, and optical polarization signature of the carbon antisite-vacancy pair in silicon carbide $(\mathrm{SiC})$. The presented theoretical study reveals the details of the physics of this important single photon source, which can serve as a flying qubit produced in quantum information processing applications.

So far this thesis has focused on the development of $\mathrm{SiC}$ and diamond based post-silicon information processing technologies. On the other hand, as we have seen in the introduction, there are numerous distinct directions as well as novel materials that are studied in this respect. As an outlook, in the second example we contribute to a very recent and fast developing field where two dimensional materials' particular band structure and the appearing quasi-particles can be utilized for advanced post-silicon information processing.

In the following sections, dominantly the group theoretical analyses are presented, the complementary theoretical and experimental studies can be found in Paper VII and Paper VIII.

\subsection{Carbon antisite-vacancy pair}

From the quantum communication and information procession point of view, the generation of flying qubits, for instance single photons, is one of the fundamental requirement, see Chapter 1. For future everyday applications, an applicable single-photon source should operate at room temperature with very high repetition 
frequency. Along this direction, an ultra bright room-temperature single-photon source in the technologically mature $4 \mathrm{H}-\mathrm{SiC}$ host is investigated experimentally and theoretically in Paper VII. The theoretical analysis includes first principles simulations as well as group theoretical description of the center's electronic structure and the selection rules of its photo luminescence transitions.

The studied centers are the so called AB lines in $4 \mathrm{H}-\mathrm{SiC}$, which were assigned to the neutral carbon antisite - carbon vacancy (CAV) complex[114]. Our calculations, however, demonstrated that no visible luminescence is possible from this configuration, thus we proposed the positively charged CAV defect as a microscopic model for the AB centers, where visible zero-phonon emission is predicted.

(a)

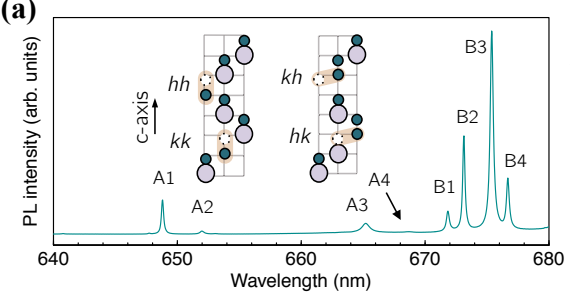

(b)
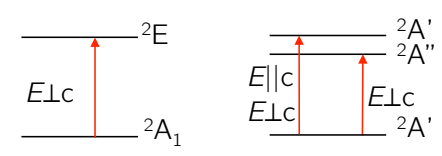

$h h, k k\left(C_{3 v}\right)$ (c)

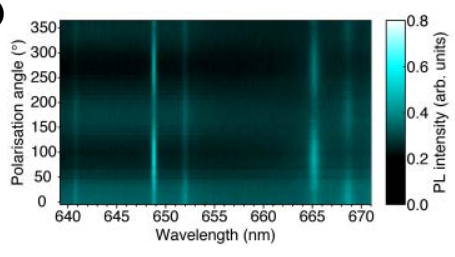

(d)

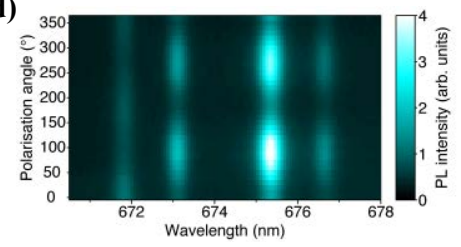

Figure 6.1. (a) The AB PL lines observed in an electron irradiated sample measured at $80 \mathrm{~K}$. The proposed atomic structure of the defects, $\mathrm{C}_{\mathrm{Si}} \mathrm{V}_{\mathrm{C}}$, giving rise to the PL, are shown in the (1120) plane with the c-axis indicated in the inset (small and large circles represent $\mathrm{C}$ and $\mathrm{Si}$ atoms while the small open circles are vacancies). The carbon antisite-vacancy pair has four possible configurations in $4 H$-SiC: $h h / k k$ axial configurations with $C_{3 v}$ symmetry, and $h k / k h$ basal plane configurations with $C_{1 h}$ symmetry. $h$ and $k$ denote the hexagonal and cubic sites in the lattice, respectively. (b) Schematic diagram of the ground-state electronic structure of axial and basal plane configurations of the positively charged carbon antisite-vacancy defect. The symmetry labels of the defect states are depicted for each configuration. Arrows represent the feasible optical transitions with the corresponding polarization of light. (c) and (d) The emission polarization dependence of the $\mathrm{AB}$ lines with the excitation beam perpendicular to the c-axis and the polarization angle $\theta$, with $\theta=0$ parallel to the c-axis. Constructed from the data presented in Paper VII.

Pair defects in 4H-SiC, such the CAV center, can have four distinguishable configurations, which are denoted as $h h, k k, h k$, and $k h$, see Fig. 6.1(a). The $h h$ and $k k$ configurations have $C_{3 v}$ symmetry, while the $h k$ and $k h$ configurations have lower $C_{1 h}$ symmetry. In $C_{3 v}$ symmetry, the four dangling bonds of the carbon and silicon atoms at the vacancy site form two fully symmetric $a_{1}$ states and a double degenerate $e$ state. According to our first principles calculations, a lower lying $a_{1}$ state, localized on the anti-site carbon's dangling bond, as well as the 
$e$ state, localized on the dangling bonds of the three silicon atoms at the carbon vacancy site, appear in the band gap of $4 \mathrm{H}-\mathrm{SiC}$. In the off-axis, $C_{1 h}$ symmetry configurations no degenerate state is possible, thus the $e$ state splits into an $a^{\prime}$ and an $a^{\prime \prime}$ states. Furthermore, the $a_{1}$ state transforms into an $a^{\prime}$ state.

In the neutral charge state of the $C_{3 v}\left(C_{1 h}\right)$ defects, two electrons occupy the states in the band gap, i.e. one occupies the lower lying $a_{1}\left(a^{\prime}\right)$ state and one occupies the $e\left(a^{\prime}\right.$ or $\left.a^{\prime \prime}\right)$ states, and form spin-1 ground state[29]. First principles calculations show, on the other hand, that the neutral CAV defect can be efficiently ionized under $2.35 \mathrm{eV}$ illumination, which was used in the experiments, thus we investigate the positive charge state in the followings.

In the ground state of the positive charge state, one electron occupies the $a_{1}$ state in $C_{3 v}$ symmetry. The single electron forms a fully symmetric ${ }^{2} A_{1}$ electronic state. In the excited state, the electron is promoted to the higher lying $e$ state and forms a ${ }^{2} E$ electronic state. The ${ }^{2} E$ state is, however, Jahn-Teller unstable, thus the excited state configuration becomes spontaneously distorted and relaxes into a $C_{1 h}$ symmetry configuration. Due to this reconstruction, the $e$ state splits into an $a^{\prime}$ and an $a^{\prime \prime}$ states, while the many-body ${ }^{2} E$ state splits into ${ }^{2} A^{\prime}$ and ${ }^{2} A^{\prime \prime}$ states. In the lower symmetry, the ground state has ${ }^{2} A^{\prime}$ character.

In order to determine the selection rules of the optical transitions, first we consider the case of the $C_{3 v}$ symmetric electronic states to better understand the experimental observations, depicted in Fig. 6.1(c) and (d). As the optical dipole operator transforms as a vector, in $C_{3 v}$ symmetry as $A_{1}+E$, the optical transition

with $\mathbf{E} \| c$ polarization transforms according to $A_{1}$, while the optical transition with $\mathbf{E} \perp c$ polarization transforms according to $E$. From the multiplications of the representations of the initial and final states and the optical transition operator, see Section 2.3.4, one can see that only the $A_{1} \times E \times E$ multiplication contains the $A_{1}$ representation, thus the optical transition has $\mathbf{E} \perp c$ polarization.

In $C_{1 h}$ symmetry the ${ }^{2} E$ excited state splits, thus two optical transitions are possible with well-distinguishable zero-phonon energies. Due to the low symmetry, the selection rules relax, i.e. the ${ }^{1} A^{\prime} \rightarrow{ }^{1} A^{\prime}$ transition allows both parallel and perpendicular polarization, while ${ }^{1} A^{\prime \prime} \rightarrow{ }^{1} A^{\prime}$ transition allows only $\mathbf{E} \perp c$ photon emission.

In conclusion, we expect two luminescence lines from each CAV configurations. As the relaxed $C_{1 h}$ structures are close to the undistorted $C_{3 v}$ symmetric configuration, we expect a stronger, perpendicularly polarized emission as well as a weaker perpendicularly or parallelly polarized emission from each centers. As these expectations are in good agreement with the experimental observations, they further confirm the microscopic model of the AB lines.

\subsection{Dirac points in novel 2D materials}

The discovery of massless Dirac particle in the band structure of graphene[115] has initiated a whole new research area, which nowadays extends to the investigation of other low dimensional systems that can host massless quasiparticles.[116] Such systems have great potential for realizing new generation of sensors, transistors 
and other information communication technologies.[117-119]

MXene's family[120] is one of the youngest among the 2D materials.[119] However, little is known about these materials, they already exhibit great potential in applications, due to the variety and richness of the different MXene phases' properties.

MXenes are produced through the chemical exfoliation[121] of bulk or thin film MAX[122] phases.[120] The obtained transition-metal (M) and carbon or nitrogen (X) compounds form 2D sheets, which have chemically active surfaces. These surfaces are supposed to be terminated by elements (fluorine and oxygen) and groups (such as hydroxil) that are present in the chemical exfoliation.[120] The passivating atomic layers bond mainly through ionic interactions, thus the valence number of the terminating elements or groups can largely affect the number of electrons remained in the MXene sheet. Consequently, by passivating the surface with different elements, the Fermi level can notable shift in the slightly perturbed band structure of the MXene sheets. Therefore, different terminations can yield different conducting properties, such as metallic or semiconducting[120, 123].

In Paper VIII, metallic MXene phases are investigated. It is shown that several of these phases can exhibit Dirac cones in their band structure with peculiar properties. Due to the strong spin-orbit interaction of the cone-forming $d$-orbital related bands, a notable gap opens at the Dirac points, which makes these systems an exciting realization of the Kane-Mele model[117], originally proposed for graphene with very small spin-orbit splitting of the Dirac point.

Among other properties of these, so called, Dirac MXenes, their band structures, particularly the Dirac cone forming bands were investigated in Paper VIII by the means of group-theoretical considerations and first principles calculations.

(a)

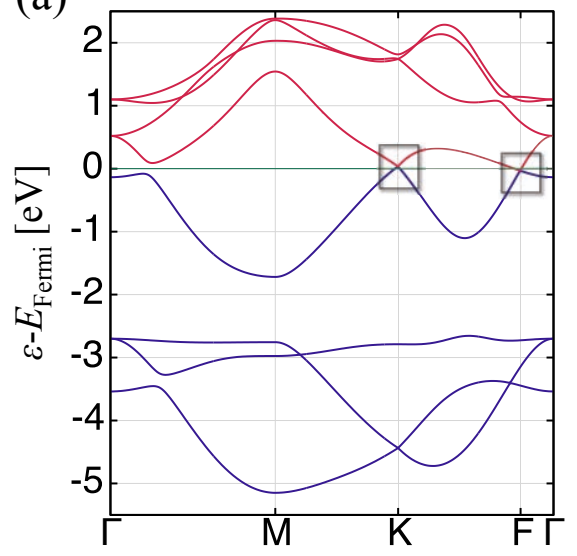

(b)

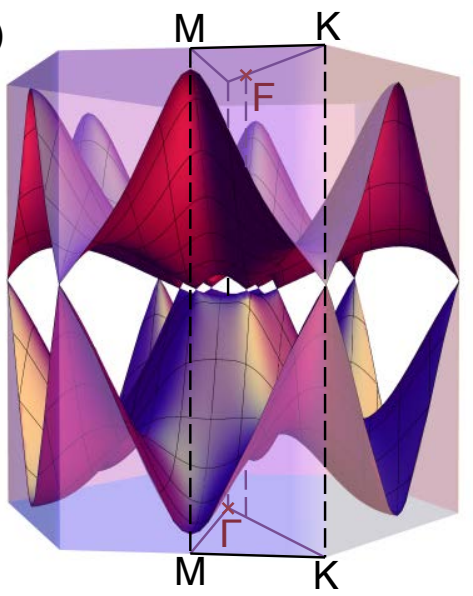

Figure 6.2. Band structure of $\mathrm{Ti}_{2} \mathrm{CF}_{2}-(\mathrm{BB})$ showing Dirac points. (a) Energy dispersion of the bands along the high symmetry lines of the two-dimensional Brillouin zone. The Fermi energy is chosen to be the zero value of the energy scale. (b) 3D plot of the cone-forming bands at the Fermi level over the 2D symmetrical Brillouin zone. Constructed from the data presented in Paper VIII. 
As can be seen in Fig. 6.2, not one but two Dirac cones were observed along the high symmetry lines of the Brillouin zone of the non-relativistic band structure of a fluorine terminated MXene sheet. One cone is located at the $K$ point, similarly to graphene, while an additional one can be found along the $K-\Gamma$ line, close to the $\Gamma$ point. The presence of this additional cone raises several questions, like: Is this indeed a cone with a single contact point or a higher dimension intersection? We apply group-theoretical considerations to answer this question.

The point group of MXene sheets with atomic termination layers on both sides is $D_{3 d}=D_{3} \otimes i$, which is a subgroup of graphene's $D_{6 d}$ point group. The point group of the wave vector at the high-symmetry point $\Gamma, K$, and $M$ is $D_{3 d}, D_{3}$, and $C_{2 h}$, along the high symmetry lines $\Gamma-K, K-M$, and $M-\Gamma$ is $C_{2}, C_{2}$, and $C_{1 v}$, and $C_{1}$ in every other nonsymmetric points of the hexagonal Brillouin zone, respectively. Among these point groups, two dimensional representation belongs only to the $D_{3 d}$ and $D_{3}$ point groups, thus two-fold degeneracy is allowed only at the $\Gamma$ and $K$ points. Consequently, essential Dirac point, claimed by the high symmetry of the wave vector, is only possible at these points. On the other hand, the Dirac point along the $\Gamma-K$ line, at the $F$ point must be accidental, in the sense that it appears due to the accidental crossing of the bands. As this point is not determined by the symmetry, it is questionable if it is a point contact or a part of a higher dimensional intersection.

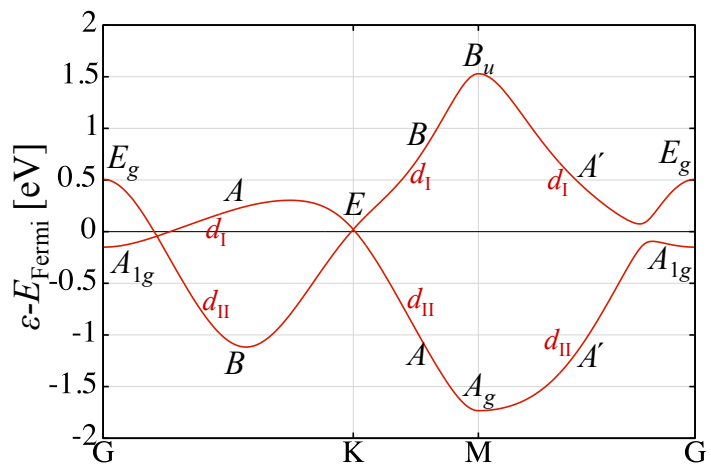

Figure 6.3. Band structure of $\mathrm{Ti}_{2} \mathrm{C}_{-} \mathrm{F}_{2}(\mathrm{BB})$ MXene sheet near the Fermi level. The point group representations are indicated. Adopted from Paper VIII.

In Fig.6.3, the point group representations of the cone-forming $d_{I}$ and $d_{I I}$ bands of the non-relativistic bands structure are presented. As can be seen, along the $\Gamma-K$ and $K-M$ lines the two bands have different representations, thus interaction cannot mix these bands. Consequently, the $d_{I}$ and $d_{I I}$ bands can cross along the $\Gamma-K$ and $K-M$ lines. On the other hand, along the $M-\Gamma$ line the bands have the same representation, thus the Coulomb interaction can mix them. In this case the bands do not cross, an avoided crossing can be observed with a finite gap. Such avoided crossing can be seen along the $M-\Gamma$ line, close to the $\Gamma$ point. Note also that the non-symmetrical wave-vectors belong to the $C_{1}$ point group, which has only one irreducible representation, thus the $d_{I}$ and $d_{I I}$ bands cannot cross in the 
non-symmetrical points of the Brillouin zone.

From the above described group-theoretical reasoning one can see that, a band crossing along the $\Gamma-K$ line forms a cone point, as in its vicinity only avoided crossing is possible. Consequently, at the $F$ point, the $d_{I}$ and $d_{I I}$ bands exhibit a Dirac point. Note, on the other hand that, as this point is formed accidentally, its position can be changed by symmetry conserving perturbations. 


\section{CHAPTER 7}

\section{Conclusions and outlook}

Within this thesis different aspects of paramagnetic point defect based quantum information processing were concerned. In connection with these aspects, different theoretical methods were developed and implemented to provide applicable tools for gaining dipper insight to related phenomena. As a results of my studies, correlated point defect in semiconductors can be described accurately, the effects of external perturbation on the zero-field-splitting of high-spin ground state defects can be better understood, study of the details of dynamic nuclear polarization has became possible, the ultrabright carbon antisite-vacancy pair single-photon emitter in silicon carbide was identified and better understood, and last but not least, Dirac-points in novel 2D materials are predicted.

Though the methods described in this thesis are connected to the area of quantum quantum information processing and communication, they have implications on other research areas too. In the following sections the perspectives of the developed methods are discussed in the respective of quantum information processing and a broader sense.

\subsection{The hybrid-DFT $+\mathrm{V}_{w}$ scheme}

The hybrid-DFT $+\mathrm{V}_{w}$ method has already demonstrated its usefulness and accuracy in the case of transition-metal impurities in conventional semiconductor hosts. However, the quantum information processing community has less interest toward transition-metal related qubits, probably due to absence of ODMR signal from such defects, accurate description of these color centers' properties is valuable for the semiconductor physics and material science communities. As transition-metal related defects introduce deep states in the band gap, they can serve as charge carrier traps and recombination centers. These properties can be utilized to fabricate 
semi-insulating samples with very short charier lifetime.

The proposed method can have its most significant implication on the application of hybrid functional on correlated materials. The derivation, which is connected to the introduction of the hybrid-DFT $+\mathrm{V}_{w}$ method, explains how the static correlation effects are included in the hybrid functionals. Although, due to the limitations of hybrids, by applying the proposed $\mathrm{V}_{w}$ correction, more accurate results could be obtained in certain cases. Additional, a possible direction of further development could be the introduction of the hybrid-DFT + DMFT method, in which both the non-correlated itinerant and correlated states could be described accurately.

\subsection{Zero-field-splitting calculation}

Beside quantum information processing applications, the zero-field-splitting tensor calculation can be used, for instance, for the identification of high-spin state point defects' configurations. To do so, convergence tests are needed to be carried out in order to reveal the factors that can limit the accuracy of the calculations. To the best of my knowledge such studies have not been published yet.

As we have seen in the application of the zero-field-splitting tensor calculation, thermal expansion only cannot account for the experimentally observed temperature dependence. By the inclusion of phonons, for instance, through ab initio molecular dynamics simulations, the calculated temperature dependent shift of the zero-field-splitting could better reproduce the experimental results.

\subsection{Dynamic nuclear spin polarization}

The model of the dynamic nuclear polarization (DNP) was used to understand the physics of the NV center in diamond and the divacancy and related centers in $\mathrm{SiC}$. On the other hand, similar models can be used for the simulation of other qubit candidates' DNP process, for instance, the DNP of silicon vacancy in SiC.

Beside quantum information processing applications, DNP has great potential in sensitivity-enhanced nuclear magnetic resonance (NMR) and magnetic resonance imagining (MRI) applications. DNP is a fast and efficient way to polarize nuclear spins close to electron spin quantum bits. Through the diffusion of the nuclear spin polarization, large number of nuclear spins can be polarized that substantially increases the efficiency of NMR and MRI measurements. As the nuclear spin - nuclear spin spin diffusion is slow compared to the DNP process, the efficiency of the bulk nuclear spin polarization could be substantially increased by direct DNP of farther, weakly coupled nuclei. The results, presented in this thesis, show that the ground state DNP of the NV center diamond or the divacancy in $\mathrm{SiC}$ can be used to polarize such farther nuclei too. 


\section{Bibliography}

[1] Andrew Targowski. Information Technology and Societal Development. Hershey, PA: IGI Global, 2009.

[2] J. Bardeen and W. H. Brattain. "The Transistor, A Semi-Conductor Triode". In: Phys. Rev. 74 (1948), pp. 230-231.

[3] William Shockley, John Bardeen, and Walter Houser Brattain. "Physics 1956". In: Physics 1942-1962. Elsevier, 2013, pp. 313 -386.

[4] Dalibor Biolek et al. "Active elements for analog signal processing: classification, review, and new proposals". In: Radioengineering 17 (2008), pp. 1532 .

[5] James L Massey. "Applied digital information theory". In: lecture notes, ETH Zurich. Available: http://www.isiweb.ee.ethz.ch/archive/massey_scr /adit1.pdf (1998).

[6] Chenming Hu. Modern Semiconductor Devices for Integrated Circuits. Prentice Hall, 2010.

[7] M.A. Nielsen and I.L. Chuang. Quantum Computation and Quantum Information. Cambridge University Press, 2000.

[8] P. W. Shor. "Algorithms for quantum computation: discrete logarithms and factoring". In: Foundations of Computer Science, 1994 Proceedings., 35th Annual Symposium. 1994, pp. 124-134.

[9] Artur Ekert and Richard Jozsa. "Quantum computation and Shor's factoring algorithm". In: Rev. Mod. Phys. 68 (1996), pp. 733-753.

[10] Nicolas Gisin et al. "Quantum cryptography". In: Rev. Mod. Phys. 74 (2002), pp. 145-195.

[11] Paul Benioff. "The computer as a physical system: A microscopic quantum mechanical Hamiltonian model of computers as represented by Turing machines". In: Journal of Statistical Physics 22 (1980), pp. 563-591. 
[12] Richard P. Feynman. "Simulating physics with computers". In: International Journal of Theoretical Physics 21 (1982), pp. 467-488.

[13] D. Deutsch. "Quantum Theory, the Church-Turing Principle and the Universal Quantum Computer". In: Proceedings of the Royal Society of London A: Mathematical, Physical and Engineering Sciences 400 (1985), pp. 97117.

[14] Lov K. Grover. "A Fast Quantum Mechanical Algorithm for Database Search". In: Proceedings of the Twenty-eighth Annual ACM Symposium on Theory of Computing. STOC '96. Philadelphia, Pennsylvania, USA: ACM, 1996, pp. 212-219.

[15] D. P. Divincenzo. "Topics in Quantum Computers". In: Mesoscopic Electron Transport. Ed. by Lydia L. Sohn, Leo P. Kouwenhoven, and Gerd Schön. Dordrecht: Springer Netherlands, 1997, pp. 657-677.

[16] T. D. Ladd et al. "Quantum computers". In: Nature 464 (2010), pp. 45-53.

[17] James N. Eckstein and Jeremy Levy. "Materials issues for quantum computation". In: MRS Bulletin 38 (2013), pp. 783-789.

[18] David D. Awschalom et al. "Quantum Spintronics: Engineering and Manipulating Atom-Like Spins in Semiconductors". In: Science 339 (2013), pp. $1174-1179$.

[19] A. Gali et al. "Theory of Neutral Divacancy in SiC: A Defect for Spintronics". In: Mater. Sci. Forum 645-648 (2010), pp. 395-397.

[20] J. R. Weber et al. "Quantum computing with defects". In: PNAS 107 (2010), pp. 8513-8518.

[21] Adam Gali. "Time-dependent density functional study on the excitation spectrum of point defects in semiconductors". In: physica status solidi (b) 248 (2011), pp. 1337-1346.

[22] A. Gali. "Excitation spectrum of point defects in semiconductors studied by time-dependent density functional theory". In: J. Mat. Res. 27 (2012), p. 897.

[23] Victor A. Soltamov et al. "Room Temperature Coherent Spin Alignment of Silicon Vacancies in 4H- and 6H-SiC". In: Phys. Rev. Lett. 108 (2012), p. 226402.

[24] H. Kraus et al. "Room-temperature quantum microwave emitters based on spin defects in silicon carbide". In: Nature Physics 10 (2014), pp. 157-162.

[25] Matthias Widmann et al. "Coherent control of single spins in silicon carbide at room temperature". In: Nat. Mater. 14 (2015), pp. 164-168.

[26] L. du Preez. PhD thesis. University of Witwatersrand, 1965.

[27] F. Jelezko and J. Wrachtrup. "Single defect centres in diamond: A review". In: Phys. Stat. Sol. A 203 (2006), p. 3207.

[28] William F. Koehl et al. "Room temperature coherent control of defect spin qubits in silicon carbide". In: Nature 479 (2011), p. 84. 
[29] Krisztián Szász et al. "Spin and photophysics of carbon-antisite vacancy defect in $4 H$ silicon carbide: A potential quantum bit". In: Phys. Rev. B 91 (2015), p. 121201.

[30] A. Sipahigil et al. "Indistinguishable Photons from Separated Silicon-Vacancy Centers in Diamond". In: Phys. Rev. Lett. 113 (2014), p. 113602.

[31] Takayuki Iwasaki et al. "Germanium-Vacancy Single Color Centers in Diamond". In: Scientific Reports 5 (2015), p. 12882.

[32] Abram L. Falk et al. "Polytype control of spin qubits in silicon carbide". In: Nature Commun. 4 (2013), p. 1819.

[33] Rogerio de Sousa and S. Das Sarma. "Theory of nuclear-induced spectral diffusion: Spin decoherence of phosphorus donors in Si and GaAs quantum dots". In: Phys. Rev. B 68, 115322 (2003), p. 115322.

[34] A. M. Tyryshkin et al. "Electron spin relaxation times of phosphorus donors in silicon". In: Phys. Rev. B 68 (2003), p. 193207.

[35] G. Balasubramanian et al. "Ultralong spin coherence time in isotopically engineered diamond". In: Nature Mater. 8 (2009), p. 383.

[36] B. B. Buckley et al. "Spin-Light Coherence for Single-Spin Measurement and Control in Diamond". In: Science 330 (2010), p. 1212.

[37] L. Robledo et al. "High-fidelity projective read-out of a solid-state spin quantum register". In: Nature 477 (2011), p. 574.

[38] David J. Christle et al. "Isolated electron spins in silicon carbide with millisecond coherence times". In: Nat. Mater. 14 (2015), pp. 160-163.

[39] Andrea Morello et al. "Single-shot readout of an electron spin in silicon". In: Nature 467 (2010), pp. 687-691.

[40] H. Büch et al. "Spin readout and addressability of phosphorus-donor clusters in silicon". In: Nat. Commun. 4 (2013), p. 2017.

[41] B.H. Bransden and C.J. Joachain. Quantum Mechanics. Pearson Education Limited, 2000.

[42] M. Born and R. Oppenheimer. "Zur Quantentheorie der Molekeln". In: Annalen der Physik 389 (1927), pp. 457-484.

[43] Felix Bloch. "Über die Quantenmechanik der Elektronen in Kristallgittern". In: Zeitschrift für Physik 52 (1929), pp. 555-600.

[44] L. H. Thomas. "The calculation of atomic fields". In: Proc. Cambridge Phil. Soc. 23 (1927), pp. 542-548.

[45] E. Fermi. "Un Metodo Statistico per la Determinazione di alcune Priopriet dell'Atomo". In: Rend. Accad. Naz. Lincei 6 (1927), 602-607.

[46] P. Hohenberg and W. Kohn. "Inhomogeneous Electron Gas". In: Phys. Rev. 136 (1964), B864-B871.

[47] W. Kohn and L. J. Sham. "Self-Consistent Equations Including Exchange and Correlation Effects". In: Phys. Rev. 140 (1965), A1133-A1138. 
[48] W. Kohn. "Nobel Lecture: Electronic structure of matter-wave functions and density functionals". In: Rev. Mod. Phys. 71 (1999), pp. 1253-1266.

[49] R. M. Martin. Electronic Structure: Basic Theory and Practical Methods. Cambridge University Press, 2004.

[50] A. Seidl et al. "Generalized Kohn-Sham schemes and the band-gap problem". In: Phys. Rev. B 53 (1996), pp. 3764-3774.

[51] Yue Wang and John P. Perdew. "Correlation hole of the spin-polarized electron gas, with exact small-wave-vector and high-density scaling". In: Phys. Rev. B 44 (1991), pp. 13298-13307.

[52] R. O. Jones. "Density functional theory: Its origins, rise to prominence, and future". In: Rev. Mod. Phys. 87 (2015), pp. 897-923.

[53] John P. Perdew, Kieron Burke, and Matthias Ernzerhof. "Generalized Gradient Approximation Made Simple". In: Phys. Rev. Lett. 77 (1996), pp. 38653868.

[54] John P. Perdew and Mel Levy. "Physical Content of the Exact Kohn-Sham Orbital Energies: Band Gaps and Derivative Discontinuities". In: Phys. Rev. Lett. 51 (1983), pp. 1884-1887.

[55] L. J. Sham and M. Schlüter. "Density-Functional Theory of the Energy Gap". In: Phys. Rev. Lett. 51 (1983), pp. 1888-1891.

[56] John P. Perdew et al. "Density-Functional Theory for Fractional Particle Number: Derivative Discontinuities of the Energy". In: Phys. Rev. Lett. 49 (1982), pp. 1691-1694.

[57] J. P. Perdew and Alex Zunger. "Self-interaction correction to densityfunctional approximations for many-electron systems". In: Phys. Rev. B 23 (1981), pp. 5048-5079.

[58] J. Harris. "Adiabatic-connection approach to Kohn-Sham theory". In: Phys. Rev. A 29 (1984), pp. 1648-1659.

[59] Axel D. Becke. "A new mixing of Hartree-Fock and local density-functional theories". In: The Journal of Chemical Physics 98 (1993), pp. 1372-1377.

[60] Stephan Kümmel and Leeor Kronik. "Orbital-dependent density functionals: Theory and applications". In: Rev. Mod. Phys. 80 (2008), pp. 3-60.

[61] Carlo Adamo and Vincenzo Barone. "Toward reliable density functional methods without adjustable parameters: The PBE0 model". In: The Journal of Chemical Physics 110 (1999), pp. 6158-6170.

[62] Jochen Heyd, Gustavo E. Scuseria, and Matthias Ernzerhof. "Hybrid functionals based on a screened Coulomb potential". In: J. Chem. Phys. 118 (2003), p. 8207.

[63] Jochen Heyd, Gustavo E. Scuseria, and Matthias Ernzerhof. "Erratum: "Hybrid functionals based on a screened Coulomb potential" [ J. Chem. Phys. 118, 8207 (2003) ]". In: J. Chem. Phys. 124 (2006), p. 219906. 
[64] Jochen Heyd and Gustavo E. Scuseria. "Efficient hybrid density functional calculations in solids: Assessment of the Heyd-Scuseria-Ernzerhof screened Coulomb hybrid functional". In: The Journal of Chemical Physics 121 (2004), pp. 1187-1192.

[65] Federico Iori, Matteo Gatti, and Angel Rubio. "Role of nonlocal exchange in the electronic structure of correlated oxides". In: Phys. Rev. B 85 (2012), p. 115129 .

[66] Sivan Refaely-Abramson et al. "Quasiparticle Spectra from a Nonempirical Optimally Tuned Range-Separated Hybrid Density Functional". In: Phys. Rev. Lett. 109 (2012), p. 226405.

[67] Viktor Atalla et al. "Hybrid density functional theory meets quasiparticle calculations: A consistent electronic structure approach". In: Phys. Rev. B 88 (2013), p. 165122.

[68] Vladimir I. Anisimov, Jan Zaanen, and Ole K. Andersen. "Band theory and Mott insulators: Hubbard $U$ instead of Stoner I". In: Phys. Rev. B 44 (1991), pp. 943-954.

[69] A. I. Liechtenstein, V. I. Anisimov, and J. Zaanen. "Density-functional theory and strong interactions: Orbital ordering in Mott-Hubbard insulators". In: Phys. Rev. B 52 (1995), R5467-R5470.

[70] V. I. Anisimov, F. Aryasetiawan, and A. I. Lichtenstein. "First-principles calculations of the electronic structure and spectra of strongly correlated systems: the LDA + U method". In: Journal of Physics: Condensed Matter 9 (1997), p. 767.

[71] S. L. Dudarev et al. "Electron-energy-loss spectra and the structural stability of nickel oxide: An LSDA+U study". In: Phys. Rev. B 57 (1998), pp. $1505-1509$.

[72] Matteo Cococcioni and Stefano de Gironcoli. "Linear response approach to the calculation of the effective interaction parameters in the LDA $+\mathrm{U}$ method". In: Phys. Rev. B 71 (2005), p. 035105.

[73] Hong Jiang et al. "First-principles modeling of localized $d$ states with the GW@LDA + U approach". In: Phys. Rev. B 82 (2010), p. 045108.

[74] V. I. Anisimov et al. "Density-functional theory and $\mathrm{NiO}$ photoemission spectra". In: Phys. Rev. B 48 (1993), pp. 16929-16934.

[75] M. T. Czy żyk and G. A. Sawatzky. "Local-density functional and on-site correlations: The electronic structure of $\mathrm{La}_{2} \mathrm{CuO}_{4}$ and $\mathrm{LaCuO}_{3}$ ". In: Phys. Rev. B 49 (1994), pp. 14211-14228.

[76] Burak Himmetoglu et al. "Hubbard-corrected DFT energy functionals: The $\mathrm{LDA}+\mathrm{U}$ description of correlated systems". In: International Journal of Quantum Chemistry 114 (2014), pp. 14-49.

[77] Marcus W. Doherty et al. "The nitrogen-vacancy colour centre in diamond". In: Physics Reports 528 (2013), pp. $1-45$. 
[78] J R Maze et al. "Properties of nitrogen-vacancy centers in diamond: the group theoretic approach". In: New Journal of Physics 13 (2011), p. 025025.

[79] Adam Gali. "Identification of individual ${ }^{13} \mathrm{C}$ isotopes of nitrogen-vacancy center in diamond by combining the polarization studies of nuclear spins and first-principles calculations". In: Phys. Rev. B 80 (2009), p. 241204.

[80] Ran Fischer et al. "Optical polarization of nuclear ensembles in diamond". In: Phys. Rev. B 87 (2013), p. 125207.

[81] G. Kresse and J. Hafner. "Ab initio molecular-dynamics simulation of the liquid-metal-amorphous-semiconductor transition in germanium". In: Phys. Rev. B 49 (1994), pp. 14251-14269.

[82] G. Kresse and J. Furthmüller. "Efficient iterative schemes for ab initio total-energy calculations using a plane-wave basis set". In: Phys. Rev. B 54 (1996), pp. 11169-11186.

[83] Vienna Ab initio Simulation Package. https://www.vasp.at/.

[84] Krisztián Szász et al. "Hyperfine coupling of point defects in semiconductors by hybrid density functional calculations: The role of core spin polarization". In: Phys. Rev. B 88 (2013), p. 075202.

[85] Mildred S. Dresselhaus, Gene Dresselhaus, and Ado Jorio. Group Theory. Springer, 2008.

[86] Lars Hedin. "New Method for Calculating the One-Particle Green's Function with Application to the Electron-Gas Problem". In: Phys. Rev. 139 (1965), A796-A823.

[87] Chris G. Van de Walle and Jörg Neugebauer. "First-principles calculations for defects and impurities: Applications to III-nitrides". In: J. Appl. Phys. 95 (2004), pp. 3851-3879.

[88] Christoph Freysoldt, Jörg Neugebauer, and Chris G. Van de Walle. "Fully Ab Initio Finite-Size Corrections for Charged-Defect Supercell Calculations". In: Phys. Rev. Lett. 102 (2009), p. 016402.

[89] M. Schulz et al. Impurities and defects in group IV elements, IV-IV and III$V$ compounds. Ed. by Lamdolt-Börnstein. Vol. 41. Berlin: Springer, 2003.

[90] W. J. Choyke, H. Matsunami, and G. Pensl, eds. Recent Major Advances in SiC. Berlin Heidelberg: Springer-Verlag, 2004.

[91] A. Gällström, B. Magnusson, and E. Janzén. "Optical Identification of Mo Related Deep Level Defect in $4 \mathrm{H}$ and $6 \mathrm{H} \mathrm{SiC".} \mathrm{In:} \mathrm{Mater.} \mathrm{Sci.} \mathrm{Forum} \mathrm{615-}$ 617 (2009), p. 405.

[92] F. C. Beyer et al. "Deep levels in tungsten doped n-type 3C-SiC". In: Appl. Phys. Lett. 98, 152104 (2011), p. 152104.

[93] John P. Perdew et al. "Exchange and correlation in open systems of fluctuating electron number". In: Phys. Rev. A 76 (2007), p. 040501.

[94] Juanita Jaramillo, Gustavo E. Scuseria, and Matthias Ernzerhof. "Local hybrid functionals". In: The Journal of Chemical Physics 118 (2003), pp. 10681073. 
[95] Aliaksandr V. Krukau et al. "Hybrid functionals with local range separation". In: The Journal of Chemical Physics 129, 124103 (2008).

[96] John P. Perdew et al. "Density-Functional Theory for Fractional Particle Number: Derivative Discontinuities of the Energy". In: Phys. Rev. Lett. 49 (1982), pp. 1691-1694.

[97] John P. Perdew and Mel Levy. "Comment on Significance of the highest occupied Kohn-Sham eigenvalue". In: Phys. Rev. B 56 (1997), pp. 1602116028.

[98] Stephan Lany and Alex Zunger. "Polaronic hole localization and multiple hole binding of acceptors in oxide wide-gap semiconductors". In: Phys. Rev. B 80 (2009), p. 085202.

[99] Ismaila Dabo et al. "Koopmans' condition for density-functional theory". In: Phys. Rev. B 82 (2010), p. 115121.

[100] M. J. Rayson and P. R. Briddon. "First principles method for the calculation of zero-field splitting tensors in periodic systems". In: Phys. Rev. B 77 (2008), p. 035119.

[101] P. E. Blöchl. "Projector augmented-wave method". In: Phys. Rev. B 50 (1994), pp. 17953-17979.

[102] G. Kresse and D. Joubert. "From ultrasoft pseudopotentials to the projector augmented-wave method". In: Phys. Rev. B 59 (1999), pp. 1758-1775.

[103] X.-D. Chen et al. "Temperature dependent energy level shifts of nitrogenvacancy centers in diamond". In: Applied Physics Letters 99, 161903 (2011).

[104] Marcus W. Doherty et al. "Electronic Properties and Metrology Applications of the Diamond NV ${ }^{-}$Center under Pressure". In: Phys. Rev. Lett. 112 (2014), p. 047601.

[105] M. W. Doherty et al. "Temperature shifts of the resonances of the NV center in diamond". In: Phys. Rev. B 90 (2014), p. 041201.

[106] N. T. Son et al. "Divacancy in 4H-SiC". In: Phys. Rev. Lett. 96 (2006), p. 055501.

[107] Xing-Fei He, Neil B. Manson, and Peter T. H. Fisk. "Paramagnetic resonance of photoexcited N- $V$ defects in diamond. I. Level anticrossing in the ${ }^{3}$ A ground state". In: Phys. Rev. B 47 (1993), pp. 8809-8815.

[108] G. Fuchs et al. "Excited-State Spectroscopy Using Single Spin Manipulation in Diamond". In: Phys. Rev. Lett. 101 (2008), p. 117601.

[109] V. Jacques et al. "Dynamic Polarization of Single Nuclear Spins by Optical Pumping of Nitrogen-Vacancy Color Centers in Diamond at Room Temperature". In: Phys. Rev. Lett. 102 (2009), p. 057403.

[110] Benjamin Smeltzer, Jean McIntyre, and Lilian Childress. "Robust control of individual nuclear spins in diamond". In: Phys. Rev. A 80 (2009), p. 050302.

[111] Benjamin Smeltzer, Lilian Childress, and Adam Gali. "13 C hyperfine interactions in the nitrogen-vacancy centre in diamond". In: New Journal of Physics 13 (2011), p. 025021. 
[112] A. Dréau et al. "High-resolution spectroscopy of single NV defects coupled with nearby ${ }^{13} \mathrm{C}$ nuclear spins in diamond". In: Phys. Rev. B 85 (2012), p. 134107.

[113] Abram L. Falk et al. "Electrically and Mechanically Tunable Electron Spins in Silicon Carbide Color Centers". In: Phys. Rev. Lett. 112 (2014), p. 187601.

[114] J. W. Steeds. "Photoluminescence study of the carbon antisite-vacancy pair in $4 H$ - and 6H-SiC". In: Phys. Rev. B 80 (2009), p. 245202.

[115] K. S. Novoselov et al. "Electric Field Effect in Atomically Thin Carbon Films". In: Science 306 (2004), pp. 666-669.

[116] T.O. Wehling, A.M. Black-Schaffer, and A.V. Balatsky. "Dirac materials". In: Advances in Physics 63 (2014), pp. 1-76.

[117] C. L. Kane and E. J. Mele. "Quantum Spin Hall Effect in Graphene". In: Phys. Rev. Lett. 95 (2005), p. 226801.

[118] Xiao-Liang Qi and Shou-Cheng Zhang. "Topological insulators and superconductors". In: Rev. Mod. Phys. 83 (4 2011), pp. 1057-1110.

[119] Ganesh R. Bhimanapati et al. "Recent Advances in Two-Dimensional Materials beyond Graphene". In: ACS Nano 9 (2015), pp. 11509-11539.

[120] Michael Naguib et al. "Two-Dimensional Nanocrystals Produced by Exfoliation of Ti3AlC2". In: Advanced Materials 23 (2011), pp. 4248-4253.

[121] Jonathan N. Coleman et al. "Two-Dimensional Nanosheets Produced by Liquid Exfoliation of Layered Materials". In: Science 331.6017 (2011).

[122] Per Eklund et al. "The Mn + 1AXn phases: Materials science and thin-film processing". In: Thin Solid Films 518 (2010), pp. 1851 -1878.

[123] Mohammad Khazaei et al. "Novel Electronic and Magnetic Properties of Two-Dimensional Transition Metal Carbides and Nitrides". In: Advanced Functional Materials 23 (2013), pp. 2185-2192. 


\section{List of included Publications}

[I] Role of screening in the density functional applied to transitionmetal defects in semiconductors

V. Ivády, I.A. Abrikosov, E. Janzén, A. Gali

Physical Review B, 87, 205201 (2013).

[II] Theoretical unification of hybrid-DFT and DFT + U methods for the treatment of localized orbitals

V. Ivády, R. Armiento, K. Szász, E. Janzén, A. Gali, I.A. Abrikosov Physical Review B, 90, 035146 (2014).

[III] Pressure and temperature dependence of the zero-field splitting in the ground state of NV centers in diamond: A first-principles study

V. Ivády, T. Simon, J.R. Maze, I.A. Abrikosov, A. Gali

Physical Review B, 90, 235205 (2014).

[IV] Theoretical model of dynamic spin polarization of nuclei coupled to paramagnetic point defects in diamond and silicon carbide V. Ivády, K. Szász, A.L. Falk, P.V. Klimov, D.J. Christle, E. Janzén, I.A. Abrikosov, D.D. Awschalom, A. Gali

Physical Review B, 92, 115206 (2015). 
[V] Optical polarization of nuclear spins in silicon carbide

A.L. Falk, P.V. Klimov, V. Ivády, K. Szász, D.J. Christle, W.F. Koehl, A. Gali, D.D. Awschalom

Physical Review Letters, 114, 247603 (2015).

[VI] High fidelity bi-directional nuclear qubit initialization in SiC

V. Ivády, P.V. Klimov, K.C. Miao, A.L. Falk, D.J. Christle, K. Szász, I.A. Abrikosov, D.D. Awschalom, A. Gali

Submitted to Physical Review Letters.

[VII] A silicon carbide room-temperature single-photon source

S. Castelletto, B.C. Johnson, V. Ivády, N. Stavrias, T. Umeda, A. Gali, and T. Ohshima

Nature Materials, 13, 151-156 (2014).

[VIII] Dirac points with giant spin-orbit splitting in the electronic structure of two-dimensional transition-metal carbides

H. Fashandi, V. Ivády, P. Eklund, A. Lloyd Spetz, M.I. Katsnelson, I.A. Abrikosov

Physical Review B, 92, 155142 (2015). 
Related, not included publications

\section{Articles:}

[1] Asymmetric split-vacancy defects in SiC polytypes: A combined theoretical and electron spin resonance study V. Ivády, A. Gällström, N.T. Son, E. Janzén, and A. Gali Physical Review Letters, 107, 195501 (2011).

[2] Electron paramagnetic resonance and theoretical studies of $\mathrm{Nb}$ in $4 \mathrm{H}$ - and $6 \mathrm{H}-\mathrm{SiC}$

N.T. Son, X.T. Trinh, A. Gällström, S. Leone, O. Kordina, E. Janzén, K. Szász, V. Ivády, and A. Gali

Journal of Applied Physics, 112, 083711 (2012).

[3] Enhancement of electron-nuclear hyperfine interaction at lattice defects in semiconducting single-walled carbon nanotubes studied by ab initio density functional theory calculations

V. Zólyomi, V. Ivády, and A. Gali

Physical Review B, 86, 235433 (2012).

[4] Electrically and mechanically tunable electron spins in silicon carbide color centers

A.L. Falk, P.V. Klimov, B.B. Buckley, V. Ivády, I.A. Abrikosov, G. Calusine, W.F. Koehl, A. Gali, and D.D. Awschalom

Physical Review Letters, 112, 187601 (2014). 
[5] Spin and photophysics of carbon-antisite vacancy defect in $\mathbf{4 H}$ silicon carbide: A potential quantum bit

K. Szász, V. Ivády, I.A. Abrikosov, E. Janzén, M. Bockstedte, and A. Gali Physical Review B, 91, 121201(R) (2015).

[6] Optical properties and Zeeman spectroscopy of niobium in silicon carbide

A. Gällström, B. Magnusson, S. Leone, O. Kordina, N.T. Son, V. Ivády, A. Gali, I.A. Abrikosov, E. Janzën, and I.G. Ivanov

Physical Review B, 92, 075207 (2015).

\section{Proceedings:}

[7] Identification of niobium in $4 \mathrm{H}-\mathrm{SiC}$ by $\mathrm{EPR}$ and ab initio studies N.T. Son, V. Ivády, A. Gali, A. Gällström, S. Leone, O. Kordina, and E. Janzén

Materials Science Forum, 717-720, 217-220 (2012).

[8] Transition metal defects in cubic and hexagonal polytypes of SiC: Site selection, magnetic and optical properties from ab initio calculations

V. Ivády, B. Somogyi, V. Zólyomi, A. Gällström, N.T. Son, E. Janzén, and A. Gali

Materials Science Forum, 717-720, 205-210 (2012).

[9] Optical properties of the niobium centre in $4 \mathbf{H}, \mathbf{6 H}$, and $15 \mathrm{R} \mathrm{SiC}$ I.G. Ivanov, A. Gällström, S. Leone, O. Kordina, N.T. Son, A. Henry, V. Ivády, A. Gali, and E. Janzén

Materials Science Forum, 740-742, 405-408 (2013).

[10] Theoretical investigation of the single photon emitter carbon antisitevacancy pair in $4 \mathrm{H}-\mathrm{SiC}$

V. Ivády, I.A. Abrikosov, E. Janzén, and A. Gali

Materials Science Forum, 778-780, 495-498 (2014). 
[11] First principles investigation of divacancy in $\mathrm{SiC}$ Polytypes for solid state qubit application

K. Szász, V. Ivády, E. Janzén, and A. Gali

Materials Science Forum, 778-780, 499-502 (2014).

[12] Optical nuclear spin polarization of divacancies in $\mathrm{SiC}$

V. Ivády, K. Szász, A.L. Falk, P.V. Klimov, D.J. Christle, W.F. Koehl, E. Janzén, I.A. Abrikosov, D.D. Awschalom, A. Gali

Materials Science Forum, 858, 287-290 (2016). 


\section{Summary of included papers}

\section{Paper I}

Theoretical unification of hybrid-DFT and DFT $+U$ methods for the treatment of localized orbitals

\section{Summary}

In this work a formal connection is derived between the hybrid functionals and the $\mathrm{DFT}+\mathrm{U}$ method for the case of localized orbitals. Through this connection, the inclusion of static correlation effects in the hybid functionals can be understood. A method, called hybrid-DFT $+\mathrm{V}_{w}$ scheme, is introduced, discussed, and analyzed in detail.

\section{Author's contribution}

I took part in planning the study, carried out the theoretical derivation, performed the first principles calculations, participated in the discussions, and wrote majority of the manuscript.

\section{Paper II}

Role of screening in the density functional applied to transition-metal defects in semiconductors

\section{Summary}

In this study, the performance of the HSE06 hybrid functional is investigated on a set of transition metal impurities in conventional semiconductor hosts. We reveal the shortcomings of the HSE06 functional and relate it to the insufficient screen- 
ing of the bare Coulomb repulsion between the localized defect states. A potential correction is proposed that could remedy the limitations of the HSE06 functional.

\section{Author's contribution}

I took part in planning the study, carried out the first principles calculations, participated in the discussions, and wrote the manuscript jointly.

\section{Paper III}

Pressure and temperature dependence of the zero-field splitting in the ground state of NV centers in diamond: A first-principles study

\section{Summary}

Within the framework of this study, a first principles zero-field-splitting tensor calculation is implemented and employed to investigate the pressure and temperature dependence of the zero-field-splitting of the NV center in diamond. The microscopic response to the applied pressure is analyzed. It is shown that thermal expansion alone cannot account for the experimentally observed temperature shift.

\section{Author's contribution}

I took part in planning the study, performed the theoretical derivations, the implementation, and the first principles calculations, participated in the discussions, and wrote majority of the manuscript.

\section{Paper IV}

Theoretical model of dynamic spin polarization of nuclei coupled to paramagnetic point defects in diamond and silicon carbide

\section{Summary}

In this work, a comprehensive theoretical model of the dynamic nuclear spin polarization (DNP) of optically polarizable paramagnetic point defects in semiconductors is developed and tested. Through this model several experimental results are understood and discussed in detail. Among others, it is demonstrated that the electron spin relaxation can have dominant effect on the efficiency of the dynamic nuclear spin polarization process. We show that it is particularly important to include spin relaxation effects in the excited state DNP of the NV center in diamond and divacancy in silicon carbide. 


\section{Author's contribution}

I took part in planning the study, carried out the theoretical derivations, performed the numerical simulations, participated in the discussions, and wrote majority of the manuscript.

\section{Paper V}

\section{Optical polarization of nuclear spins in silicon carbide}

\section{Summary}

In this work highly efficient dynamic nuclear spin polarization is experimentally demonstrated by divacancy defects in silicon carbide.

\section{Author's contribution}

I performed the theoretical simulations, explained some the observations, participated in the discussions, and contributed to the manuscript.

\section{Paper VI}

\section{High fidelity bi-directional nuclear qubit initialization in $\mathrm{SiC}$}

\section{Summary}

In this study the ground state dynamic nuclear polarization of paramagnetic point defects is theoretically and experimentally investigated. It is shown by theory that weakly coupled nuclear spin-point defect spin hybrid systems can be polarized with high efficiency, while the nuclear polarization can exhibit a sensitive magnetic field dependent inversion. A similar effect is theoretically predicted and experimentally confirmed for the case of a strongly coupled hybrid spin system in silicon carbide.

\section{Author's contribution}

I took part in planning the study, carried out the theoretical study and the numerical simulations, participated in the discussions, and wrote majority of the manuscript. 


\section{Paper VII}

\section{A silicon carbide room-temperature single-photon source}

\section{Summary}

In this work a remarkably bright room-temperature single-photon emitter is investigated in silicon carbide by both experimental and theoretical means. With the help of the theoretical analysis, the microscopic structure of this important single-photon source is revealed.

\section{Author's contribution}

I performed the theoretical simulations, participated in the theoretical analysis, participated in the discussions, and contributed to the manuscript.

\section{Paper VIII}

Dirac points with giant spin-orbit splitting in the electronic structure of two-dimensional transition-metal carbides

\section{Summary}

In this study the properties of metallic two-dimensional transition-metal carbides, the so called MXenes, are investigated by theoretical means. We demonstrate that several phases' band structure possesses Dirac cones close to the Fermi level. The described Dirac fermions' physics exhibits important differences compared to graphene's massless electrons. For instance, an outstandingly large spin-orbit splitting is predicted at the Dirac points.

\section{Author's contribution}

I took part in planning the study, carried out part of the first principles calculations and the theoretical analysis, participated in the discussions, and wrote the paper jointly. 


\section{Papers}

The articles associated with this thesis have been removed for copyright reasons. For more details about these see:

http://urn.kb.se/resolve?urn=urn:nbn:se:liu:diva-131853 